The Version of Record of this manuscript has been published and is available in

Journal of Mathematics and Music, March 2020

https://doi.org/10.1080/17459737.2020.1726690

\title{
An alternative approach to generalized Pythagorean scales. Generation and properties derived in the frequency domain
}

\author{
Rafael Cubarsi \\ Departament de Matemàtiques, Universitat Politècnica de Catalunya, Barcelona, Spain
}

\begin{abstract}
scales are formalized as a cyclic group of classes of projection functions related to iterations of the scale generator. Their representatives in the frequency domain are used to built cyclic sequences of tone iterates satisfying the closure condition. The refinement of cyclic sequences with regard to the best closure provides a constructive algorithm that allows to determine cyclic scales avoiding continued fractions. New proofs of the main properties are obtained as a consequence of the generating procedure. When the scale tones are generated from the two elementary factors associated with the generic widths of the step intervals we get the partition of the octave leading to the fundamental Bézout's identity relating several characteristic scale indices. This relationship is generalized to prove a new relationship expressing the partition that the frequency ratios associated with the two sizes composing the different step-intervals induce to a specific set of octaves.
\end{abstract}

Keywords: well-formed scales; equal temperament; Pythagorean tuning; Pythagorean comma; Bézout's identity; continued fractions

2010 Mathematics Subject Classification: 05C12; 11A05; 54E35

\section{Introduction}

An $n$-tone equal temperament (TET) scale does not include the pitch classes (PCs) of the harmonics and subharmonics of a fundamental frequency, not even the lowest orders, but in its favour its PCs remain equally spaced within the circle of the octave. The scale intervals have an algebraic structure similar to the group of integers modulo $n,\left(\mathbb{Z}_{n},+\right)$, and any PC may assume the role of the fundamental. On the other hand, a just intonation scale composed of a finite family of harmonics and subharmonics, apart from having non-equal temperament, is not closed with regard to the composition of its intervals. A compromise is reached in Pythagorean tuning, where the scale is tuned to the class of the third harmonic, the lowest harmonic not belonging to the class of the fundamental. The standard 12-tone Pythagorean scale gives an acceptable approximation of the harmonics up to the tenth-except the seventh that sounds very low in most instruments (von Helmholtz 1863)- and is close to a 12-TET scale with an error smaller than one Pythagorean comma. For these reasons, Pythagorean tuning has become the standard pattern of Western music. By choosing conveniently the number $n$ of notes, it is possible to get a Pythagorean scale containing notes closer to the lower harmonics. On the other hand, it is well known that the frequency ratios between the scale tones are aurally significant since the harmonic structure of many musical instruments causes their partials to overlap. For this reason, in order to produce specific overtone series, spectrum, or timbre (Sethares 1998), Pythagorean tuning can be generalized to scales generated by intervals other than the fifth.

While trying to substantiate the process of building a Pythagorean scale, one finds that the properties inherent to the formation of the scale do not depend on the generator interval chosen,

Email: rafael.cubarsi@upc.edu 
rather they are a consequence of the process itself. Hence, as already suggested by Regener (1967), the procedure can be extended to musical scales generated as a finite sequence of iterations of an arbitrary frequency ratio $h$ (with regard to a fundamental $\nu_{0}=1$ ). Since the octave is associated with a frequency ratio 2, the reduction of the frequencies by octaves to the interval $[1,2)$ determines the representatives of the frequency classes (FCs), which will be referred to as scale tones. When one iterate is closer than the others to either end of the interval, the scale formed up to this iterate satisfies the closure condition (Carey and Clampitt 1989). Since the reference octave is usually represented as a circle in the pitch domain $\left(\log _{2}\right.$ of the frequencies), we will say the scale is cyclic, meaning a well-formed scale of one generator, which will be described as a cyclic group. In the general case, $h$ is assumed to be different from a rational power of 2 , otherwise the scale is equally tempered, which is considered as a degenerate Pythagorean system. The procedure can be generalized by changing the period of the octave to any positive value $\omega$.

In the current paper, cyclic scales will be studied by following such a constructive procedure, by pointing out, especially in the footnotes, the links to other approaches, namely the continued fractions approximation of $\log _{2} h$, combinatorics of words, Farey sums, or the Stern-Brocot tree (Carey and Clampitt 2012, 2017; Clampitt and Noll 2011; Noll 2006, 2007, 2008, 2015; Jedrzejewski 2009, 2008). After introducing the notation, an abstract scale is described from a canonical Generalized Interval System (GIS) (Lewin 1987) with an interval function, that we call projection function, related to iterations of the generator tone. Under an equivalence relation, the representatives of the classes of projection functions define the tones of a cyclic scale. Hereafter, we leave the abstract formulation and adopt a more analytical viewpoint. In a first instance, we present an approach leading for efficiently determining these tones. It is worked out in the frequency domain instead of the pitch domain, as usual. The reason is to show in what simple way a cyclic scale can be generated: (a) there is an infinite hierarchy of cyclic sequences increasing the number of tones; (b) each sequence is characterized by the minimum and maximum tones $\nu_{m}$ and $\nu_{M}$, ordered as increasing pitches in $(1,2)$; (c) since $\frac{\nu_{M}}{2}<1<\nu_{m}$, then $\frac{\nu_{M}}{2}<\frac{\nu_{M} \nu_{m}}{2}<\nu_{m}$, from where we prove that a new extreme tone of the next cyclic sequence must be $\nu_{m+M}$. By identifying this tone of the sequence with the fundamental we get the cyclic scale. In this way, we obtain alternative proofs to results usually derived from the continued fractions approach (e.g., Carey and Clampitt 1989; Hellegouarch 1999; Douthett and Krantz 2007). Later on, the generating algorithm is reformulated at a generic level depending exclusively on the indices of the scale tones, without the need to estimate the comma (e.g., Noll 2007). In this form, the algorithm allows to distinguish easily between optimal scales (associated with the convergents of the continued fractions approach) and non-optimal scales (associated with the semi-convergents).

In a second part, new proofs of several scale properties are derived from the generating procedure. The paper provides new and alternative viewpoints to obtain the main characterizations of well-formed scales, such as those related to the best comma (Douthett and Krantz 2007), the chromatic length of the generator, Myhill's property (Clough and Myerson 1985, 1986), the spectrum width, etc. As a new result we prove that, for a $n$-tone cyclic scale, the difference between the chromatic length of the generating ratio $N$ and the index of octave $\llbracket n$ (octave windings in $n$ ) tells whether the scale is or is not optimal.

Finally, we study the partition of the octave. After providing a new demonstration of how the frequency ratios associated with the extreme tones (i.e., those corresponding to the two possible sizes of the step-interval $j=1$ ) generate the partition of the octave into elementary factors such that $\nu_{m}^{M}\left(\frac{2}{\nu_{M}}\right)^{m}=2$, we generalize it. Thus, if the pair $\nu_{l}, \frac{2}{\nu_{L}}$, with $l+L=n$, is the spectrum of the $j$-th step-interval, then we get $\nu_{l}^{L}\left(\frac{2}{\nu_{L}}\right)^{l}=2^{j}$, which provides the partition of $j$ octaves and yields the generic relationship between the indices $j, l, L$. Generating the cyclic scale from such a pair of factors with indices coprime with $n$, which in the $\log _{2}$ domain is equivalent to the pairs generator/co-generator, gives rise to self-similar pitch structures representing the scale (Carey and Clampitt 1996; Clampitt and Noll 2011). In addition. it provides the deviation of the scale tones with regard to the $n$-TET scale. 


\section{Preliminaries}

\subsection{Well-formed scales}

Well-formed scales satisfy the symmetry condition, consisting of displaying several degrees of rotational symmetry, which Carey and Clampitt (1989) proved to be equivalent to the closure condition. Zabka $(2009,2010)$ generalized this result and proved the equivalence of the closure and symmetry conditions also for scales generated by two intervals. In the current paper, cyclic scale refers to a well-formed scale with one generator. If one wishes a particular frequency ratio $h$ to be the generator of a non-degenerate cyclic scale, i.e., reproducing the closure and symmetry conditions, the cardinal $k$ of its pitch class (PC) within the scale must be coprime with $n$. For generalized Pythagorean systems, well-formed scales are also known as moment of symmetry (MOS) (Wilson 1975) and natural scales (Hellegouarch 1999). We highlight the three main approaches leading to cyclic scales.

The first one is the MOS (Wilson 1974, 1975), which results from iterating a non-degenerate generalized Pythagorean system when the partition of the octave induced by the scale notes has exactly two sizes of scale steps and each number of generic intervals occurs also in two different sizes $^{1}$. This condition is known as Myhill's property (Clough and Myerson 1985, 1986).

Alternatively, Carey and Clampitt (1989) founded well-formed Pythagorean scales on two theorems (Carey and Clampitt 2012, 2017). According to the first theorem, these scales have a number of PCs corresponding to the denominators of convergent and semi-convergent continued fraction expansions of $\log _{2} 3$. These numbers are associated with intervals that contain no pitches that occur earlier in the sequence of fifths (primary intervals). In other words, the span of the closure interval (i.e., the number of steps between the first and last note of the scale when the scale notes are ordered by pitch) is 1 . The properties of the continued fraction convergents determine automorphisms that rearrange the fifths iterations according to the symmetry condition. The second theorem states that these scales satisfy Myhill's property.

Another approach (Hellegouarch 1999; Kassel and Kassel 2010) determines successive natural scales from a quotient group $G / H$, where $G$ is the group generated by the frequency ratios 2 and 3 , and $H$ is the group generated by the comma $r_{n}$. If $\frac{p_{n}}{q_{n}}$ is a convergent or semi-convergent of the continued fraction expansion of $\log _{2} 3$, the comma $r_{n}=\left(\frac{3^{q_{n}}}{2^{p_{n}}}\right)^{(-1)^{n}}$ satisfies $r_{n} \rightarrow 1$ as $\frac{p_{n}}{q_{n}} \rightarrow \log _{2} 3$. The representative of each $\mathrm{PC}$ is chosen as the simplest irreducible fraction allowing an isomorphism $\varphi$ between the $q_{n}$ tones in $[1,2)$ and the set $\left\{0,1, \ldots, q_{n}-1\right\}$ so that $\varphi(2)=q_{n}$. However, for three or more generators, Žabka (2010) proved that the generalization of Hellegouarch's natural scales produces scales that are not necessarily well-formed.

From a mathematical viewpoint, the present paper integrates aspects of all the above approaches, in addition to providing new points of view. In the frequency domain, cyclic scales are associated with one of the extreme tones providing a best approximation to the frequency class 1, while optimal scales are associated with the best comma (Douthett and Krantz 2007). When the last tone of a cyclic sequence is identified with the fundamental, a structure of cyclic group arises, which representatives in $[1,2)$ define a cyclic scale.

\subsection{Frequency classes}

We first introduce the notation by reviewing the basic notion of pitch class. For any frequency ratio $\nu \in \Omega \equiv(0, \infty)$, all the values $2^{k} \nu, k \in \mathbb{Z}$, define one equivalence class $\bar{\nu}$. A ratio of 2 between values corresponds to the frequency range of one octave. The set $\Omega$ is a commutative group for $\times$. The set of all the octaves of the fundamental frequency ratio $\left(\nu_{0}=1\right)$ is a monogenous subgroup of $\Omega$ of infinite cardinal, $\Omega^{2}=\left\{2^{k}, k \in \mathbb{Z}\right\}$.

\footnotetext{
${ }^{1}$ This is a case of a maximally even set, where each generic interval is either a single integer or two consecutive integers (Clough and Douthett 1991).
} 
The frequency classes (FCs) are the elements of the quotient group $\Omega_{0}=\Omega / \Omega^{2}$, also commutative for multiplication. For each equivalence class, we choose a representative tone in $[1,2)$, the reference octave, which we identify with $\Omega_{0}$. Throughout the current paper, frequency classes will be denoted with these representatives. The FC of $\nu \in \Omega$ is determined as $\bar{\nu}=2^{\left\{\log _{2} \nu\right\}} \in \Omega_{0}$, where the curly brackets mean the fractional part. The integer part $z=\left\lfloor\log _{2} \nu\right\rfloor$ (expressed in terms of the floor function) determines the octave $\Omega_{z}=\left[2^{z}, 2^{z+1}\right.$ ) where $\nu$ is set. Thus, $\nu=2^{z} \bar{\nu}$.

Alternatively, each FC $\bar{\nu} \in \Omega_{0}$ is associated with a $\mathrm{PC}\left\{\log _{2} \bar{\nu}\right\}$ in the circle of the octave $S_{0}=\mathbb{R} / \mathbb{Z}$. Therefore, the multiplicative structure of $\Omega_{0}$ becomes an additive structure in $S_{0}$, where notes and intervals are represented.

We shall now generalize and formalize the operation of obtaining FCs, since our purpose is to obtain the best approximations in the multiplicative space of the frequency domain, instead of the additive space of their logarithms. Given the frequency ratio $\alpha \in \Omega$, the function ${ }^{2}$ that maps any frequency ratio $\beta \in \Omega$ to the $\mathrm{FC} \overline{\alpha \beta} \in \Omega_{0}$ is defined as

$$
\pi_{\alpha}: \Omega \rightarrow \Omega_{0}, \quad \pi_{\alpha} \beta=\overline{\alpha \beta} .
$$

It will be referred to as projection function of step $\alpha$, or $\alpha$-projection function. It is surjective. The identity, $\pi_{1}$, applied to $\alpha$ returns its $\mathrm{FC} \pi_{1} \alpha=\bar{\alpha}$. It satisfies $\pi_{\bar{\alpha}}=\pi_{\alpha}$. Therefore, we may write $\pi_{\frac{3}{2}}$ instead of $\pi_{3}$, or $\pi_{\frac{4}{3}}$ instead of $\pi_{\frac{1}{3}}$ and $\pi_{3^{-1}}$, regardless whether the step belongs to $\Omega$ or $\Omega_{0}$.

The composition of projection functions is well defined from the product of FCs. It satisfies

$$
\pi_{\alpha} \pi_{\beta} 1=\pi_{\beta} \pi_{\alpha} 1=\pi_{\alpha \beta} 1
$$

We can then identify the FC $\overline{\alpha \beta}$ with the projection function $\pi_{\alpha \beta}$, by assuming it is applied to 1. If we restrict the previous function to the domain of FCs $\Omega_{0}$, for any $\alpha \in \Omega$ the function

$$
\pi_{\alpha}: \Omega_{0} \rightarrow \Omega_{0}, \quad \pi_{\alpha} \beta=\overline{\alpha \beta}
$$

is bijective. Then, the family of projection functions is an Abelian group for composition, isomorphic with the group $\Omega_{0}$.

We say that $\alpha$ and $\beta$ belong to complementary classes $^{3}$ if they satisfy $\pi_{\alpha} \pi_{\beta}=\pi_{1}$. Then $\pi_{\alpha^{-1}}=\pi_{\beta}$. Hence, $\pi_{\alpha}^{-1}=\pi_{\alpha^{-1}}$. The $\alpha$-projection function is equivalent to the inverse function whose step is the complementary $\mathrm{FC}$ of $\alpha, \pi_{\alpha}=\pi_{\alpha^{-1}}^{-1}$. In general, for $n \in \mathbb{Z}$, we have $\pi_{\alpha}^{n}=\pi_{\alpha^{n}}$, although it is not true for non-integer exponents.

\subsection{One-generator sets}

We focus on the non-trivial case of the family generated by a frequency ratio $\alpha \in \Omega$ other than an integer power of 2 . That is, the set defined as $\Omega^{\alpha}=\left\{\alpha^{k}, k \in \mathbb{Z}\right\}$. This family is an infinite cyclic subgroup of $\Omega$ and its FCs are the elements of the quotient group $E^{\alpha} \equiv \Omega^{\alpha} / \Omega^{2}$, isomorphic to the family of projection functions generated by $\pi_{\alpha}$. Unless explicitly specified, we shall identify the FC $\overline{\alpha^{k}}$ to the projection function $\pi_{\alpha}^{k}$. Thus, we write

$$
E^{\alpha}=\left\{\pi_{\alpha}^{k}, k \in \mathbb{Z}\right\}
$$

\footnotetext{
${ }^{2}$ In a more formal context, we consider a GIS (Lewin 1987; Kolman 2004) $\pi: \Omega \times \Omega \rightarrow \Omega_{0}$ and a canonical GIS $\pi: \Omega_{0} \times \Omega_{0} \rightarrow \Omega_{0}$, with the $\alpha$-projection function defined as $\pi_{\alpha}(\cdot) \equiv \pi(\alpha, \cdot)$.

${ }^{3}$ Two FCs $\alpha$ and $\beta$ other than 1 are complementary if they satisfy $\alpha \beta=2$. This definition is not valid for the FC 1 .
} 
As a particular case of Dirichlet's approximation theorem ${ }^{4}$, if $\alpha \neq 2^{r}, r \in \mathbb{Q}$, then $E^{\alpha}$ is an infinite monogenous subgroup of $\Omega_{0}$, whose FCs form a dense subset. Otherwise, if $\alpha=2^{\frac{m}{n}}$, with $\frac{m}{n} \in \mathrm{Q}$ an irreducible fraction, we get a cyclic group of order $n$, which describes a $n$-TET scale that we shall write as

$$
E_{n}^{\top}=\left\{\pi_{2^{1 / n}}^{k}, k \in \mathbb{Z}\right\}
$$

although it suffices to take into account only the values $k=0, \ldots, n-1$.

We may also consider subfamilies of $E^{\alpha}$ with regard to a particular set of indices $A$, namely $E_{A}^{\alpha}=\left\{\pi_{\alpha}^{k}, k \in A\right\}$. In a general case, $E_{A}^{\alpha}$ is not a subgroup of $E^{\alpha}$ unless $A$ is a subgroup of $\mathbb{Z}$, i.e., $A=n \mathbb{Z}$, with $n \in \mathbb{N}$. However, the only case leading to a finite cyclic group is that of equation (5), since the subgroups of $\mathbb{Z}$ are of infinite cardinal. In particular, when $A=\{1, \ldots, n\}$ we will rank the elements of $E_{A}^{\alpha}$ as a $n$-order sequence

$$
S_{n}^{\alpha}=\left(\pi_{\alpha}^{k}\right)_{k=1, \ldots, n} .
$$

Notice that a sequence $S_{n}^{\alpha}$ does not begin with $\pi_{\alpha}^{0}=1$. It starts with the index $k=1$. In addition, it is not closed for composition of FCs. Only if $\alpha=2^{\frac{m}{n}}$, with $m, n$ coprime positive integers, is $S_{n}^{\alpha}$ a finite cyclic group, which is $E_{n}^{\top}$, and the value $k=n$ yields $\pi_{2}=1$.

\subsection{Algebraic structure}

The process of considering that a particular FC $\pi_{\alpha}^{n}$ plays the same role as the class of the fundamental can be summarized as follows. Several projection function iterates $\pi_{\alpha}^{0} \equiv 1, \ldots, \pi_{\alpha}^{n}$, corresponding to FCs in $\Omega_{0}$, are generated by choosing $n$ such that, in the circle of the octave $S_{0}$, the shortest interval between $\pi_{\alpha}^{n}$ and 1 , of length ${ }^{5} \epsilon=d\left(\pi_{\alpha}^{n}, 1\right)$ and corresponding to a frequency ratio $\kappa=2^{\epsilon}$, contains no previous iterates. The quantity $\kappa$ generalizes the Pythagorean comma. Then, in the family of projection functions $E^{\alpha}$ we define an equivalence relation identifying two projection functions $\pi_{\alpha}^{p}, \pi_{\alpha}^{q}$ whenever there exists an integer $r$ such that $\pi_{\alpha}^{p}=\pi_{\alpha}^{q} \pi_{\alpha}^{r n}$.

The classes of projection functions are obtained by considering the subgroup $E^{\alpha^{n}}=\left\{\pi_{\alpha}^{k n}, k \in\right.$ $\mathbb{Z}\} \subset E^{\alpha}$ and the quotient group

$$
\bar{E}_{n}^{\alpha} \equiv E^{\alpha} / E^{\alpha^{n}}=\left\{\bar{\pi}_{\alpha}^{k}, k \in \mathbb{Z}_{n}\right\} .
$$

Therefore, $\bar{E}_{n}^{\alpha}$ is a finite cyclic group. For each class $\bar{\pi}_{\alpha}^{k}$, we shall choose a representative $\pi_{\alpha}^{k}$ satisfying $0 \leq k<n$ (since we are interested in generalizing the Pythagorean tuning, where the PCs are obtained by applying successive fifths to the fundamental ratio 1).

Nevertheless, alternative scales can be obtained by choosing an other representative of the class. For instance, Hellegouarch (1999) constructs an abstract scale as a factor group (see also Kassel and Kassel 2010). Starting from a comma $\kappa$, he defines a Pythagorean scale as the one generated from the harmonic of order 3 , in addition to the second harmonic determining the octave, modulo the group generated by comma, i.e., $\langle 2,3\rangle /\langle\kappa\rangle$. In such a case, the representative of the class is chosen as the tone given by the irreducible fraction $\frac{p}{q}$ with $\max (p, q) \operatorname{minimal}^{6}$. In the end, it comes down to choosing either the projection function $\pi_{\alpha}^{k}$ or $\pi_{\alpha}^{k-n}$, so that some tones

\footnotetext{
${ }^{4}$ For any $x \in \mathbb{R}$ and any positive $N \in \mathbb{Z}$, there exists integers $p$ and $q$ such that $1 \leq q \leq N$ and $|q x-p|<\frac{1}{N}$.

5 The shortest distance between two FCs $\alpha, \beta$ is measured in the unit circle $S_{0}$ as $d(\alpha, \beta)=\min \left(\left|\log _{2} \frac{\alpha}{\beta}\right|, 1-\left|\log _{2} \frac{\alpha}{\beta}\right|\right)$. For a FC $\pi_{\alpha}^{k} \in(1,2)$ we have $d\left(\pi_{\alpha}^{k}, 1\right)=\min \left(\log _{2} \pi_{\alpha}^{k}, 1-\log _{2} \pi_{\alpha}^{k}\right)$, while for its complementary FC, $\pi_{\alpha}^{-k}=\frac{2}{\pi_{\alpha}^{k}}$, we also have $d\left(\pi_{\alpha}^{-k}, 1\right)=\min \left(1-\log _{2} \pi_{\alpha}^{k}, \log _{2} \pi_{\alpha}^{k}\right)$. Thus, $d\left(\pi_{\alpha}^{k}, 1\right)=d\left(\pi_{\alpha}^{-k}, 1\right)$.

${ }^{6}$ In our approach, the scale remains composed of tones corresponding to positive powers of 3 , and the 12-tone Pythagorean scale is formed by successive fifths of 1 ; while for these authors a natural scale is also composed by tones expressed as negative powers of 3 , so that the Pythagorean scale is formed by successive fifths of $\frac{256}{243}$.
} 
are obtained as positive powers and others as negative. By this method, these authors determine the optimal scales (continued fractions convergents).

Our procedure has also important similarities with Regener's (1973) approach. His interval transformations provide a primary instance of Generalized Interval System (Lewin 1987) in the special case of notes and note intervals in mediation with frequencies and frequency ratios. Focusing in pitch notation, he also constructs a music scale as a factor group of octave and comma classes, which he call enharmonic lines (Regener 1973, p. 106).

The group structure of $\left(\bar{E}_{n}^{\alpha}, \cdot\right)$ for composition is obviously isomorphic to $\left(\mathbb{Z}_{n},+\right)$. The representatives of the classes of projection functions applied to the fundamental determine $n$ tones in $\Omega_{0}$ that define an $\alpha$-cyclic scale, according to

$$
E_{n}^{\alpha}=\left\{\pi_{\alpha}^{k} ; k=0, \ldots, n-1\right\} .
$$

Since the abstract scale $\bar{E}_{n}^{h}$ is isomorphic to the finite cyclic group $\mathbb{Z}_{n}$, any class $\bar{\pi}_{h}^{p}$ with $p \in \mathbb{Z}_{n}$ coprime with $n$ generates the scale ${ }^{7}$. In Appendix A we briefly review how to deal with cyclic scales from an arithmetical point of view.

In the limiting case $\pi_{\alpha}^{n} \rightarrow 1$ we get an equal temperament scale, i.e., a degenerate cyclic scale. Therefore, the better the approximation $\pi_{\alpha}^{n} \approx 1$, the closer $E_{n}^{\alpha}$ to a $n$-TET scale,

$$
\pi_{\alpha}^{n} \rightarrow 1 \Rightarrow E_{n}^{\alpha} \rightarrow E_{n}^{\top}
$$

A precise quantification will be done. For this reason we could say that a non-degenerate $\alpha$-cyclic scale $E_{n}^{\alpha}$ is a scale asymptotically equally tempered, with $\pi_{\alpha}^{n}$ converging to 1 when $n$ increases to $\infty$.

\section{Cyclic sequences and scales}

Let us examine a novel way to build cyclic sequences and scales and the important role played by the extreme tones (after arranging the scale tones in pitch order and excluding the fundamental). The immediate generalization of a Pythagorean scale is a scale generated by a harmonic of the fundamental $h \in \mathbb{Z}^{+}$(coprime with 2). However, the structure of these scales is the same for any value $h \in \mathbb{R}^{+}$. Furthermore, there is no loss of generality by assuming the scale is generated by a representative $h \in(1,2)$ of a FC. For a non-degenerate scale, first we find out the tones generated by the projection $\pi_{h}$, i.e., $\pi_{h}^{p}=\overline{h^{p}}, p \in \mathbb{Z}$. Since $\pi_{h}^{p} \in \Omega_{0}$, we write $\pi_{h}^{p}=\frac{h^{p}}{2^{q}}, \quad 1 \leq \frac{h^{p}}{2^{q}}<2$. Then, by multiplying by $2^{q}$ and taking logarithms to the base 2 , we get $q \leq p \log _{2} h<q+1$, hence, $p \log _{2} h-1<q \leq p \log _{2} h$. Therefore, $q$ is univocally determined from $p$ as the following integer part

$$
q=\llbracket p \rrbracket \equiv\left\lfloor p \log _{2} h\right\rfloor .
$$

Given $h$, the tones resulting from applying iteratively the projection $\pi_{h}$ become expressed as

$$
\nu_{p} \equiv \pi_{h}^{p}=\frac{h^{p}}{2 \llbracket p \rrbracket}, \quad p \in \mathbb{Z} .
$$

\footnotetext{
${ }^{7}$ It is worth remembering that $\mathbb{Z}_{n}$ has the same structure of commutative ring than the integers for the addition and product. In the ring $\mathbb{Z}_{n}$ of integers modulo $n, 1$ is the neutral element for the product. The invertible elements form the multiplicative group of units $U\left(\mathbb{Z}_{n}\right)$. Therefore, $a \in \mathbb{Z}_{n}$ has inverse if and only if there is $b \in \mathbb{Z}_{n}$ such that $a b=1$, hence $a, b \in U\left(\mathbb{Z}_{n}\right)$. Similarly, two integers $x, y \in \mathbb{Z}$, such that $x y \equiv 1(\bmod n)$, belong to two classes of $U\left(\mathbb{Z}_{n}\right)$ that are mutually inverse, or belong to the class of 1 . This condition can be written using Bézout's identity. This leads to the property that the generators of $\left(\mathbb{Z}_{n},+\right)$, which are coprime with $n$, are just the elements of $U\left(\mathbb{Z}_{n}\right)$.
} 
The value $\llbracket p \rrbracket$ is the index of octave of $h^{p}$, i.e., the number of the octave $\Omega_{\llbracket p \rrbracket}$ where the tone $h^{p}$ lies. To simplify, we may read $\llbracket p \rrbracket$ as octave of $p$. For positive integers, if $p^{\prime}>p$ then $p^{\prime} \log _{2} h>p \log _{2} h$ and $\left\lfloor p^{\prime} \log _{2} h\right\rfloor \geq\left\lfloor p \log _{2} h\right\rfloor$. Therefore, we get ${ }^{8} p^{\prime}>p \Longrightarrow \llbracket p^{\prime} \rrbracket \geq \llbracket p \rrbracket$.

Depending on whether we take positive or negative indices in equation (11) the resulting scales are not the same, since the way of closing the cycle of fifths about the fundamental ratio 1 is different in each case. If in one case it is closed by default, in the other it is by excess. In general, positive indices will be used.

According to equation (11), since $\log _{2} h$ is irrational, the sequence $S_{n}^{h}=\left(\nu_{k}\right)_{k=1, \ldots, n}$ is never closed and the iterations $\nu_{k}$ are not ordered along the octave $\Omega_{0}$ in terms of $k$. If the tones of a sequence $S_{n}^{h}$ are ordered from the lowest to the highest pitch, in $(1,2)$ we find two extreme tones

$$
\nu_{m}=\min _{\nu \in S_{n}^{h}} \nu, \quad \nu_{M}=\max _{\nu \in S_{n}^{h}} \nu .
$$

We call them minimum and maximum tones of the sequence. Obviously, any tone $\nu_{i} \in S_{n}^{h}$ satisfies $1<\nu_{m} \leq \nu_{i} \leq \nu_{M}<2$. The indices of the extreme tones $m$ and $M$ are usually referred to as the generic widths of the step intervals. Their music-theoretical interpretation and historical traces can be found in Clampitt and Noll (2011).

In order to get the last iteration close to the fundamental, a sequence $S_{n}^{h}$ should end when $\nu_{n}$ is one of the extreme tones $\nu_{m}$ or $\nu_{M}$, so that the last iteration is closer to 1 or 2 than the previous iterations. This is a condition equivalent to that of Carey and Clampitt's (1989) primary intervals. In such a case we say that the sequence is cyclic. Then, the approximation $\nu_{n} \rightarrow 1^{+}$or $\nu_{n} \rightarrow 2^{-}$justifies the equivalence relation $\nu_{n} \sim \nu_{0}$ yielding the cyclic scale of equation (8).

\subsection{Optimal sequences and scales}

We say the cyclic sequence $S_{n}^{h}$ is optimal if the last term of the sequence is the best approximation of the fundamental. Such a distance corresponds to a frequency ratio $\kappa_{n}$, referred to as $n$-order comma,

$$
\kappa_{n}=\min \left(\nu_{n}, \frac{2}{\nu_{n}}\right)
$$

Then, the $n$-order comma is a value greater than one. Remark that the comma is not defined as the frequency ratio of the smallest distance between the FCs of the sequence and the fundamental. For this reason, we will call marginal ratios to the values $\nu_{m}$ and $\frac{2}{\nu_{M}}$, and absolute ratio of the sequence, $\rho\left(S_{n}^{h}\right)$, to their minimum, corresponding to the minimum comma of the sequence. If the sequence $S_{n}^{h}=\left(\nu_{1}, \cdots, \nu_{n}\right)$ is optimal, then $\rho\left(S_{n}^{h}\right)=\kappa_{n}$ and by identifying the tones $\nu_{n}$ and $\nu_{0}$ we get the $n$-tone optimal scale $E_{n}^{h}=\left\{\nu_{0}, \nu_{1}, \ldots, \nu_{n-1}\right\}$. The tones other than the fundamental of the optimal scale $E_{n}^{h}$ match those of the sequence $S_{n-1}^{h}$.

An optimal scale remains characterized in terms of the extreme tones according to one of the following two cases:

(i) If the tone $\nu_{n}$ is identified with the fundamental 1 from above, then the comma is $\kappa_{n}=\nu_{n}$ and the tones satisfy the conditions

$$
\begin{array}{ll}
\text { (1) } 1<\kappa_{n}=\nu_{n}<\nu_{m} \leq \nu_{k}, & k<n \\
\text { (2) } 1<\kappa_{n}=\nu_{n}<\frac{2}{\nu_{M}} \leq \frac{2}{\nu_{k}}, & k<n
\end{array} .
$$

However, if the scale is non-optimal only condition (1) is satisfied.

8 If $x, y \in \mathbb{R}$ we have $\lfloor x\rfloor+\lfloor y\rfloor \leq\lfloor x+y\rfloor \leq\lfloor x\rfloor+\lfloor y\rfloor+1$. If $h \in(1,2)$, then $\llbracket 1 \rrbracket=\left\lfloor\log _{2} h\right\rfloor=0$ and the value $\llbracket p+1 \rrbracket$ matches either $\llbracket p \rrbracket$ or $\llbracket p \rrbracket+1$. 
(ii) If the tone $\nu_{n}$ is identified with the fundamental 2 from below, the comma is $\kappa_{n}=\frac{2}{\nu_{n}}$ and the tones satisfy the conditions

$$
\begin{array}{ll}
\text { (1) } 1<\kappa_{n}=\frac{2}{\nu_{n}}<\nu_{m} \leq \nu_{k}, & k<n \\
\text { (2) } 1<\kappa_{n}=\frac{2}{\nu_{n}}<\frac{2}{\nu_{M}} \leq \frac{2}{\nu_{k}}, & k<n
\end{array} .
$$

However, if the scale is non-optimal, only condition (2) is satisfied.

\subsection{Refinement of sequences and scales}

We enumerate some immediate properties and consequences of the definition of cyclic sequences and scales, not necessarily optimal.

(1) Given a sequence $S_{n}^{h}$, if $m, M \leq n$ are the indices of the extreme tones satisfying equation (12), then $S_{m}^{h}$ and $S_{M}^{h}$ are two cyclic sequences contained in $S_{n}^{h}$. By taking $n^{\prime}=$ $\max (m, M), S_{n^{\prime}}^{h}$ is the greater cyclic sequence contained in $S_{n}^{h}$.

(2) Any pairs of cyclic sequences $S_{n^{\prime}}^{h}, S_{n}^{h}$ and scales $E_{n^{\prime}}^{h}, E_{n}^{h}$ with $n^{\prime}<n$ satisfy $S_{n^{\prime}}^{h} \subset S_{n}^{h}$ and $E_{n^{\prime}}^{h} \subset E_{n}^{h}$. We say that $S_{n}^{h}$ and $E_{n}^{h}$ are refinements of (or finer than) $S_{n^{\prime}}^{h}$ and $E_{n^{\prime}}^{h}$, respectively.

(3) The definition of extreme tones is also valid for scales, by leaving aside the fundamental ratio $\nu_{0}=1$.

(4) If $S_{n}^{h}$ is a cyclic sequence and the sequence $S_{n-1}^{h}$, not necessarily cyclic, has extreme tones $\nu_{m}=\min _{\nu \in S_{n-1}^{h}}(\nu)$ and $\nu_{M}=\max _{\nu \in S_{n-1}^{h}}(\nu)$; then, the index $n^{\prime}=\max (m, M)$ determines a cyclic sequence $S_{n^{\prime}}^{h} \subset S_{n}^{h}$ among which there is no intermediate cyclic sequence. We then say that they are consecutive cyclic sequences.

The algorithm we use to generate cyclic scales is based on the following simple fact:

$$
\frac{\nu_{M}}{2}<1<\nu_{m} \Longrightarrow \frac{\nu_{M}}{2}<\frac{\nu_{M} \nu_{m}}{2}<\nu_{m} .
$$

Let us remember that the tone $\nu_{M+m}$, as a representative of a $\mathrm{FC}$, takes the value $\frac{\nu_{M} \nu_{m}}{2}$ if $\nu_{M} \nu_{m}>2$, or $\nu_{M} \nu_{m}$ if $\nu_{M} \nu_{m}<2$. This leads to the following results (since these results, although derived from a new perspective, are equivalent to those of well-formed scales (e.g., Carey and Clampitt 1989), their proof is not provided):

THEOREM 3.1 If a sequence $S_{n}^{h}$ (not necessarily cyclic) has extreme tones of indices $m, M$, then the sequence $S_{M+m}^{h}$ improves at least one of the marginal ratios of $S_{n}^{h}$ and is cyclic.

Thus, the last tone $\nu_{M+m}$ is a new extreme tone of the sequence $S_{M+m}^{h}$, although if $S_{n}^{h}$ is optimal, $S_{M+m}^{h}$ is not necessarily optimal.

The second fact in which the generating algorithm is based can be enunciated as follows.

THEOREM 3.2 None of the marginal ratios of a cyclic sequence $S_{n}^{h}$ with indices of the extreme tones $m, M$ can be improved with a sequence of an order lower than $M+m$.

COROLlaRY 3.3 In order to determine optimal sequences it suffices to consider refinements of consecutive cyclic sequences in the form $\ldots \subset S_{n}^{h} \subset S_{M+m}^{h} \subset \ldots$

\section{3. $\quad$ Next and previous cyclic sequences and scales}

Let $S_{n}^{h}$ be a cyclic sequence with $n=\max (m, M)$. According to what has been explained above, the next cyclic sequence containing $S_{n}^{h}$, denoted as $S_{n^{+}}^{h}$, is obtained by taking $n^{+}=M+m$. The cyclic sequence previous to $S_{n}^{h}$, denoted as $S_{n^{-}}^{h}$, is also well determined. The sequence $S_{n}^{h}$ 
has maximum and minimum tones of indices $m$ and $M$. Let us write $n=\max (m, M)$ and $n^{\prime}=\min (m, M)$. Then, one extreme tone still is $n^{\prime}$, while the other is, by construction, $n-n^{\prime}$. Thus, $n^{-}=\max \left(n-n^{\prime}, n^{\prime}\right) ; \quad n=\max (m, M), n^{\prime}=\min (m, M)$. In such a way, consecutive cyclic sequences can be chained according to $S_{n^{-}}^{h} \subset S_{n}^{h} \subset S_{n^{+}}^{h}$. Since each cyclic sequence generates a cyclic scale, they are also chained as $E_{n^{-}}^{h} \subset E_{n}^{h} \subset E_{n^{+}}^{n^{+}}$.

Let us consider the cyclic sequence $S_{n^{-}}^{h}$, with extreme tones of indices $m$ and $M$, and its next sequence $S_{n}^{h}$ by application of the refinement algorithm, with $n=m+M$. Then we get the scale $E_{n}^{h}$ with indices of the extreme tones $m$ and $M$, i.e., the same that the sequence $S_{n-}^{h}$ already had. Therefore, for the scale $E_{n}^{h}$, we have $\nu_{m}=\min _{\nu \in E_{n}^{h} \backslash\{1\}}(\nu), \quad \nu_{M}=\max _{\nu \in E_{n}^{h} \backslash\{1\}}(\nu)$. These indices are associated with cyclic scales contained in $E_{n}^{h}$, i.e.,

$$
\begin{aligned}
& m<M \Longrightarrow E_{m}^{h} \subset \cdots \subset E_{M}^{h} \subset E_{n}^{h} \\
& M<m \Longrightarrow E_{M}^{h} \subset \cdots \subset E_{m}^{h} \subset E_{n}^{h}
\end{aligned}
$$

The two cyclic scales on the right-hand side are, in each case, consecutive. The previous properties allow us to implement an algorithm to refine optimal scales that is detailed in Appendix B. Although derived in an independent way, it is equivalent to a similar algorithm reasoned in terms of PCs and primary intervals given by Carey (1998, p. 88), which, on the other hand, is a formalization of the process explained in Carey and Clampitt (1989) of how a musician can obtain well-formed scales without using continued fractions. Our algorithm performs, in the frequency domain, a strategy similar to that of the Farey sums (Farey 1816), so that if two rational approximations satisfy $\log _{2} h \in\left(\frac{a^{\prime}}{a}, \frac{b^{\prime}}{b}\right)$ then we can lessen the size of the interval from a new approximation $\frac{a^{\prime}+b^{\prime}}{a+b}$ that substitutes the appropriate extreme. From another perspective, it also reproduces the structure of the Stern-Brocot tree ${ }^{9}$ (e.g., Graham, Knuth, and Patashnik 1994).

The approach is also valid for non-natural scales with any positive, real generator interval, i.e., by defining an octave-like as $\Omega / \Omega^{\omega}$, with $\omega \in \Omega$, instead of $\Omega / \Omega^{2}$.

\section{Scale properties}

\subsection{Closure and "digit" of scale}

For an optimal scale $E_{n}^{h}$, depending on whether $\nu_{n}$ is closer to 1 or 2, the equations (14) and (15), corresponding to the cases (i) and (ii) of Section 3.1, are satisfied. Since the above two cases will appear repeatedly hereafter, when a property is only valid for optimal scales it will be indicated with the subscript "opt", otherwise it will be valid for all cyclic scales.

Instead of the comma, a non-degenerate cyclic scale is better characterized by the parameter

$$
\gamma_{n}=\frac{\nu_{m} \nu_{M}}{2}=\frac{h^{m+M}}{2 \llbracket m \rrbracket+\llbracket M \rrbracket+1}
$$

that will be referred to as scale closure ${ }^{10}$. We shall see that it is a measure of several qualities, such as the closeness to the fundamental of the closure, the comma, the spectrum width, etc.

\footnotetext{
${ }^{9}$ The Stern-Brocot tree is a full binary tree where the nodes are labelled in such a way that each positive rational number occurs exactly once. Vertically, it provides the usual ordering of the rationals. For $\frac{p_{1}}{q_{1}}$ and $\frac{p_{2}}{q_{2}}$, the mediant is the fraction $\frac{p_{1}+p_{2}}{q_{1}+q_{2}}$ whose parents they are. Every row consists of the fractions that are mediants of elements of previous rows. Positive irrational numbers can be associated with a unique infinite pathway down the tree and the nodes which are passed by on such a finite or infinite path are called semi-convergents of the corresponding rational or irrational number.

${ }^{10}$ The scale closure is an intrinsic parameter of the scale. However, comparing between scales according to the closeness of the closure to 1 (its logarithm closer to 0 ) is actually equivalent to evaluating the best comma as defined in Douthett and Krantz (2007).
} 
Owning to equation (16) this quotient satisfies $\frac{\nu_{M}}{2}<\gamma_{n}<\nu_{m}$.

According to the definition of comma, we have

$$
\begin{aligned}
& \text { (i) } \gamma_{n}>1 \Longleftrightarrow \kappa_{n}=\nu_{n}=\gamma_{n} \\
& \text { (ii) } \gamma_{n}<1 \Longleftrightarrow \kappa_{n}=\frac{2}{\nu_{n}}=\gamma_{n}^{-1} .
\end{aligned}
$$

Hence, the scale closure $\gamma_{n}$ informs us whether the sequence $S_{n}^{h}$ closes just above $1\left(1<\gamma_{n}<\sqrt{2}\right)$ or just below $2\left(\frac{1}{\sqrt{2}}<\gamma_{n}<1\right)$, whereas the comma $\kappa_{n}$ always produces a value greater than 1 and does not provide such information. Therefore,

$$
\log _{2} \kappa_{n}=\left|\log _{2} \gamma_{n}\right|
$$

hence, $\log _{2} \gamma_{n}$ multiplied by 1200 gives the distance from $\gamma_{n}$ to 1 measured in cents $^{11}$. The exponent of the denominator of equation $(18)$, when $h \in(1,2)$, is the chromatic length of the generator,

$$
N=\llbracket m \rrbracket+\llbracket M \rrbracket+1
$$

since, as we shall see later, this is the number of step-intervals ${ }^{12}$ of the generating tone when the scale notes are arranged in pitch order. Thus, the scale closure is equivalent to

$$
\gamma_{n}=\frac{h^{n}}{2^{N}}
$$

Hence, we have

$$
\begin{aligned}
& \text { (i) } \gamma_{n}>1 \Longleftrightarrow 1<\gamma_{n}<\nu_{m} \Longleftrightarrow 1<\frac{2}{\nu_{M}}<\nu_{m} \\
& \text { (ii) } \gamma_{n}<1 \Longleftrightarrow \frac{\nu_{M}}{2}<\gamma_{n}<1 \Longleftrightarrow 1<\nu_{m}<\frac{2}{\nu_{M}}
\end{aligned} \text {. }
$$

Therefore, the scale closure indicates which of the ratios $\frac{2}{\nu_{M}}$ or $\nu_{m}$ is closer to 1, i.e., which of the extreme tones is closer to the fundamental. If the scale is non-optimal it only informs on the error about the fundamental associated with the last iteration $\nu_{n}$ of the sequence. For optimal scales, the ordering established in equations (14) and (15) can be more specific yet, such as,

$$
\begin{aligned}
& \text { (i) })_{\text {opt }} \gamma_{n}>1 \Longleftrightarrow \frac{1}{\nu_{m}}<\frac{\nu_{M}}{2}<\frac{1}{\gamma_{n}}<1<\gamma_{n}<\frac{2}{\nu_{M}}<\nu_{m} \\
& \text { (ii) })_{\text {opt }} \quad \gamma_{n}<1 \Longleftrightarrow \frac{\nu_{M}}{2}<\frac{1}{\nu_{m}}<\gamma_{n}<1<\frac{1}{\gamma_{n}}<\nu_{m}<\frac{2}{\nu_{M}}
\end{aligned}
$$

Notice that the index of octave $\llbracket n \rrbracket$, which determines the tone

$$
\nu_{n}=\frac{h^{n}}{2 \llbracket n \rrbracket}
$$

indicates the octave $\Omega_{\llbracket n \rrbracket}$ where the frequency $h^{n}$ is located. Now we can also give significance to $N$, since $2^{N}$ is the beginning of the octave closest to $h^{n}$. In other words, the chromatic length of

\footnotetext{
${ }^{11}$ It will be notated as $\phi\left(\gamma_{n}\right)=1200 \log _{2} \gamma_{n}$, corresponding to the width of the spectrum associated with the frequency ratios $\frac{2}{\nu_{M}}$ and $\nu_{m}$ (e.g., Douthett and Krantz 2007).

${ }^{12}$ Since $\frac{1}{\sqrt{2}}<\gamma_{n}<\sqrt{2}$, then $-\frac{1}{2}<n \log _{2} h-N<\frac{1}{2}$, and $N<n \log _{2} h+\frac{1}{2}<N+1$. Hence, by taking integer parts, we get $N \leq\left\lfloor n \log _{2} h+\frac{1}{2}\right\rfloor<N+1$, from where $N=\left\lfloor n \log _{2} h+\frac{1}{2}\right\rfloor$. Usually, the chromatic length $N$ is defined in this way. However, in the current paper such a relationship is not of particular interest. Instead, we are interested in the dependency of the scale properties on the indices $m, M$, and their respective octaves.
} 
the generator indicates how many full octaves would cover the scale $E_{n}^{h}$ if the frequencies were not reduced to FCs in the reference octave $\Omega_{0}$. By comparing equation (22) and equation (25), we have ${ }^{13}$

$$
\begin{aligned}
& \text { (i) } \gamma_{n}>1 \Longleftrightarrow 1<\nu_{n}<\nu_{m} \Longleftrightarrow N=\llbracket n \rrbracket \\
& \text { (ii) } \gamma_{n}<1 \Longleftrightarrow \nu_{M}<\nu_{n}<2 \Longleftrightarrow N=\llbracket n \rrbracket+1
\end{aligned} \text {. }
$$

Then, it is natural to define a scale digit indicating whether $N$ and $\llbracket n \rrbracket$ match or differ,

$$
\delta=N-\llbracket n \rrbracket
$$

so that, according to equation (26), we have the following equivalences

$$
\begin{aligned}
& \text { (i) } \gamma_{n}>1 \Longleftrightarrow \delta=0 \\
& \text { (ii) } \gamma_{n}<1 \Longleftrightarrow \delta=1
\end{aligned}
$$

By comparing equation (22) and equation (25), we can express the scale closure as

$$
\gamma_{n}=2^{-\delta} \nu_{n}
$$

\subsection{Pseudo-complementary classes}

Among the tones composing the scale $E_{n}^{h}$ we do not find their own complementary tones. The complementary of $\nu_{k} \neq 1$ is

$$
\nu_{-k}=\frac{2}{\nu_{k}}=\pi_{h}^{-k}=\pi_{1 / h}^{k}, k \in \mathbb{Z}
$$

which does not belong to $E_{n}^{h}$. Let us remark that, in terms of classes of projection functions, with indices in $\mathbb{Z}_{n}$, we have $\bar{\pi}_{h}^{-k}=\bar{\pi}_{h}^{n-k}$, so that

$$
\bar{\pi}_{h}^{n-k} \bar{\pi}_{h}^{k}=\bar{\pi}_{h}^{0}, k \in \mathbb{Z}_{n}
$$

However, as projection functions, with indices in $\mathbb{Z}$, the tones $\pi_{h}^{n-k}=\nu_{n-k} \in E_{n}^{h}$ and $\pi_{h}^{k}=\nu_{k}$ satisfy $\pi_{h}^{n-k} \pi_{h}^{k}=\pi_{h}^{n}$. Therefore, as projection functions, this establishes $\pi_{h}^{n-k} \pi_{h}^{k}=\nu_{n}, k \in \mathbb{Z}$. This equality, according to equation (29), can be expressed as

$$
\pi_{h}^{n-k} \pi_{h}^{k}=2^{\delta} \gamma_{n}, k \in \mathbb{Z}
$$

Then, the tones $\nu_{n-k}, \nu_{k} \in E_{n}^{h}$ are not complementary. We shall prove that these tones satisfy

$$
\nu_{n-k} \nu_{k}=2 \gamma_{n} ; \quad k=1, \ldots, n-1 .
$$

THEOREM 4.1 The scale closure can be computed from any pair of tones other than the fundamental with symmetric indices in $\mathbb{Z}_{n}$, i.e.,

$$
\gamma_{n}=\frac{\nu_{n-k} \nu_{k}}{2} ; \quad k=1, \ldots, n-1
$$

\footnotetext{
${ }^{13}$ We know that, if $k<n$ then $\llbracket k \rrbracket \leq \llbracket n \rrbracket$. Nevertheless, if $1<h<2$, in case (i) we have $\nu_{n}<\nu_{n-1}$ and $\nu_{n}=\frac{h}{2} \nu_{n-1}$, therefore $\llbracket n \rrbracket=\llbracket n-1 \rrbracket+1$; hence, $\llbracket k \rrbracket \leq \llbracket n-1 \rrbracket=\llbracket n \rrbracket-1$. Instead, in case (ii), $\nu_{n-1}<\nu_{n}$ and $h \nu_{n-1}=\nu_{n}$, therefore $\llbracket n-1 \rrbracket=\llbracket n \rrbracket$; hence, $\llbracket k \rrbracket \leq \llbracket n-1 \rrbracket=\llbracket n \rrbracket$. Thus, in both cases, $\llbracket k \rrbracket \leq N-1$ for $0 \leq k \leq n-1$.
} 

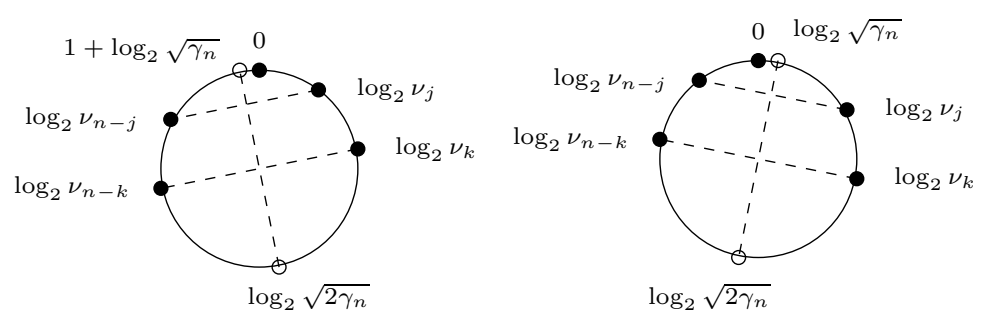

Figure 1. Symmetrical distribution of the scale notes depending on whether $\gamma_{n}$ is smaller (left) or greater (right) than 1.

Proof. In effect, equation (31), written as tones, should have the form

$$
\nu_{n-k} \nu_{k}=2^{\alpha} \gamma_{n}
$$

for some $\alpha \in \mathbb{Z}$. Bearing in mind that $\nu_{n-k}, \nu_{k} \in(1,2)$, we have $\nu_{m}<\nu_{n-k} \nu_{k}<2 \nu_{M}$. If this equation is multiplied by $2^{-\alpha}$, owing to equation (34) $\alpha$ must satisfy $2^{-\alpha} \nu_{m}<\gamma_{n}<2^{2-\alpha} \frac{\nu_{M}}{2}$. Nevertheless, on the one hand we get $\gamma_{n}<\nu_{m} \Rightarrow \alpha>0$, and on the other hand $\frac{\nu_{M}}{2}<\gamma_{n} \Rightarrow \alpha<2$. Hence, it must be $\alpha=1$ in equation (34).

If $\gamma_{n} \rightarrow 1$, the above tones are nearly complementary ${ }^{14}, \nu_{n-k} \nu_{k} \rightarrow 2$ for $k=1, \ldots, n-1$. We shall say that the tones $\nu_{n-k}$ and $\nu_{k}$ are pseudo-complementary. Also, by taking logarithms in equation (33), we get several ways to express the chromatic length $N$.

COROLlaRY 4.2 The chromatic length $N$ of the generator satisfies

$$
N=\llbracket n-k \rrbracket+\llbracket k \rrbracket+1 ; \quad k=1, \ldots, n-1 .
$$

COROLlary 4.3 In the circle of the octave $S_{0}$, the notes of pseudo-complementary tones are symmetric with regard to a diameter passing through the following points (Figure 1): one on the opposite side of the fundamental, $\log _{2} \sqrt{2 \gamma_{n}}$, and the other close to the fundamental, $\log _{2} \sqrt{\gamma_{n}}$ if $\gamma_{n}>1$ or $\log _{2} 2 \sqrt{\gamma_{n}}$ if $\gamma_{n}<1$.

Therefore, the intervals between the scale notes spread symmetrically by both sides around the point $\log _{2} \sqrt{2 \gamma_{n}}$, nearly opposite to the fundamental, and the only two non-symmetrical intervals are the two on both sides of the fundamental. The twist of the symmetry axis originates Myhill's property.

\subsection{Myhill's property}

More generally, we may analyse the relation between two classes of projection functions $\bar{\pi}_{h}^{p}$ and $\bar{\pi}_{h}^{p-k}(0<k<n)$ associated with frequency ratios between scale tones differing in a step-interval corresponding to $k h$-iterates. To this purpose, consider the equation $\bar{\pi}_{h}^{p}=\bar{\pi}_{h}^{p-k} \bar{\pi}_{h}^{\alpha}$. It can be interpreted in terms of their projection function representatives as follows:

If $p-k>0$, then $\pi_{h}^{p}=\pi_{h}^{p-k} \pi_{h}^{\alpha}$, so that $\pi_{h}^{\alpha}=\pi_{h}^{k}$.

If $p-k<0$, it is equivalent to $\pi_{h}^{p}=\pi_{h}^{n+p-k} \pi_{h}^{\alpha}$, so that $\pi_{h}^{\alpha}=\pi_{h}^{k-n}=\pi_{1 / h}^{n-k}$.

\footnotetext{
${ }^{14}$ According to equations (30) and (32), the complementary tones of the scale (other than the fundamental) are given by $\nu_{-k}=\gamma_{n}^{-1} \nu_{n-k}$, which do not match the tones of the scale $E_{n}^{h}$. The inverse scale $E_{n}^{1 / h}$, built by starting at the fundamental by negative iterations of the $h$-interval, does not provide the same scale as $E_{n}^{h}$. However, since the tones of $E_{n}^{h}$ and $E_{n}^{1 / h}$ differ by excess or by default in one comma, they belong to the same classes of projection functions. It happened in a similar way with Hellegouarch's (1999) natural scales, that were a particular choice of representatives of the abstract scale $\bar{E}_{n}^{h}$.
} 
Both projection functions $\pi_{h}^{k}$ and $\pi_{1 / h}^{n-k}$ belong to the class $\bar{\pi}_{h}^{k}$, and they correspond to the two frequency ratios $\pi_{h}^{k}=\nu_{k}$ and $\pi_{1 / h}^{n-k}=\frac{2}{\nu_{n-k}}$, which are associated with the same step-interval $k$. This demonstrates Myhill's property, by associating two possible sizes with one step-interval of the scale. For each step-interval $k$, the pair $\left(\nu_{k}, \frac{2}{\nu_{n-k}}\right)$ in the $\log _{2}$ space is referred to as the spectrum. These sizes are the respective distances from the fundamental to the two symmetric points in the graph of Figure 1. Each line connecting these two points represents a level (from top to bottom) corresponding to a generic interval. Well-formed scales remain characterized from the precisely two sizes composing the constant spectrum width $\left|\log _{2} \gamma_{n}\right|$ for the different step-intervals (Carey and Clampitt 1989, 2012, 2017), as equation (33) proves.

\subsection{Approximations of $\log _{2} h$}

For any $h>0$, if a cyclic sequence $S_{n}^{h}$ closes near the fundamental with indices $n, N$ satisfying $\frac{h^{n}}{2^{N}} \approx 1$, then $\frac{N}{n} \approx \log _{2} h$. Without loss of generality we may assume that $n$ and $N$ are coprime. In addition, if $\frac{N}{n} \approx \log _{2} h$, then $2^{\frac{N}{n}} \approx h$. Thus, by defining $\mu=N \bmod n$, the class of the generator interval is $\bar{h} \approx 2^{\frac{\mu}{n}}$, corresponding to the cardinal $\mu$. Since we had assumed that the scale generator satisfies $1<h<2$, then $\mu=N$ (in such a case, these two values $N, n$ are referred to as chromatic length of the generating interval and chromatic cardinality of the scale). Therefore, a pair $(n, N)$ associated with a cyclic scale provides a rational approximation of $\log _{2} h$ and, in addition, an equal temperament scale $E_{n}^{\top}$ whose tone $2^{\frac{N}{n}}$ is an approximation of the generator FC $h$.

More precisely, the above approximations can be described according to the following results, by relating our approach to that of continued fractions. In Appendix $\mathrm{C}$ there is a summary of the concepts hereafter involved. Since these are familiar results to music theorists (for a summary, e.g., Douthett and Krantz 2007), we will not give proofs.

Lemma 4.4 A tone $\nu_{n}$ near the fundamental provides a rational approximation of $\log _{2} h$, which is either $\frac{\llbracket n \rrbracket}{n} \rightarrow \log _{2} h^{-}$if $\nu_{n} \rightarrow 1^{+}$, or $\frac{\llbracket n \rrbracket+1}{n} \rightarrow \log _{2} h^{+}$if $\nu_{n} \rightarrow 1^{-}$.

THEOREM 4.5 The tone $\nu_{n}$ of an optimal cyclic sequence $S_{n}^{h}$ provides the best rational approximation ${ }^{15}$ of $\log _{2} h$.

In the non-optimal case: if in equation (14) only condition (1) is fulfilled, then the convergence to $\log _{2} h$ is from below; if in equation (15) only condition (2) is fulfilled, then the convergence is from above ${ }^{16}$.

Hence, according to equation (26), for cyclic scales with closure $\gamma_{n}=\frac{h^{n}}{2^{N}}$, we have:

COROLlaRY 4.6 The best estimations $\gamma_{n} \approx 1$ provide the best rational approximations $\frac{N}{n}$ of $\log _{2} h$, i.e., the convergents of its continued fraction expansion.

In a similar way, we can see how the generator interval $h$ is approximated by the power $2^{\frac{N}{n}}$.

COROLlaRY 4.7 The best estimations $\frac{2^{\frac{N}{n}}}{h} \approx 1$ provide the good rational approximations $\frac{N}{n}$ of

\footnotetext{
${ }^{15}$ The terms "good" and "best" approximation, used in recent books such as Loya (2017), are equivalent to best approximation "of the first kind" and "of the second kind", respectively, used in Khinchin (1964).

${ }^{16}$ For the cyclic scale $E_{n}^{h}$, with extreme tones of indices $m$ and $M$, the value $n$ provides a convergent or semi-convergent $\frac{N}{n}$ of $\log _{2} h$ according to one of these situations: $\frac{\llbracket m \rrbracket}{m}<\frac{N}{n}<\log _{2} h<\frac{\llbracket M \rrbracket+1}{M}$ or $\frac{\llbracket m \rrbracket}{m}<\log _{2} h<\frac{N}{n}<\frac{\llbracket M \rrbracket+1}{M}$, depending on whether $\nu_{n}$ is a new minimum or maximum of the sequence $S_{n}^{h}$. We shall see in Section 6.2 that equations (45) and (46) are satisfied. Therefore, we meet a situation such as $\frac{a^{\prime}}{a}<\log _{2} h<\frac{b^{\prime}}{b}$, together with the Bézout's identity corresponding to pairs of coprime numbers, $a b^{\prime}-b a^{\prime}=1$, which leads to a new improvement $\frac{a^{\prime}+b^{\prime}}{a+b}$ of the approximation. This situation is common to the continued fractions approach, the Farey sums, the structure of the Stern-Brocot tree, and its dual, the Raney tree (Berstel and de Luca 1997; Raney 1973), also known as Calkin-Wilf tree (Calkin and Wilf 2000; Gibbons, Lester, and Bird 2006). Thus, for two consecutive approximations, by using the extended Euclidean algorithm (Appendix D) it is possible from one to determine the other.
} 
$\log _{2} h$.

In summary, good rational approximations of $\log _{2} h$ correspond to cyclic scales, close to $n$-TET scales, that include optimal cyclic scales obtained from the best approximations. Furthermore, there are cyclic scales not associated with good or best rational approximations still corresponding to semi-convergents (see Table B1).

\section{Alternative algorithm}

Scales can be ordered according to decreasing values of $\left|\log _{2} \gamma_{n}\right|$; however, we are going to determine the chain of finer cyclic scales, and in particular the optimal ones, without needing to evaluate the comma. Instead, the scale digit $\delta$ will determine the optimal scales.

\subsection{Previous and next scale}

Starting from the indices of the extreme tones $(m, M)$ in $E_{n}^{h}$ we will calculate the same values in the scale $E_{n^{+}}^{h}$ for the next cyclic scale in the chain. For the scale $E_{n}^{h}$, with $n=m+M$, the value $\delta$, defined in equation (27), according to equation (28) tells us whether the $\mathrm{FC} \nu_{n}$, not belonging to the scale $E_{n}^{h}$, is the new minimum or maximum tone of the next scale $E_{n^{+}}^{h}$.

Then, the next improvement of the scale takes place according to one of the following cases:

(i) $\quad m^{+}=m+M, M^{+}=M ; \quad \llbracket m^{+} \rrbracket=\llbracket m \rrbracket+\llbracket M \rrbracket+1, \llbracket M^{+} \rrbracket=\llbracket M \rrbracket \quad \Longleftrightarrow \delta=0$

(ii) $m^{+}=m, M^{+}=m+M ; \quad \llbracket m^{+} \rrbracket=\llbracket m \rrbracket, \llbracket M^{+} \rrbracket=\llbracket m \rrbracket+\llbracket M \rrbracket \quad \Longleftrightarrow \delta=1$

\subsection{Matrix notation}

The equalities in equation (36) can be written in matrix form to obtain the indices of the subsequent scales as

$$
\left(\begin{array}{cc}
m^{+} & M^{+} \\
\llbracket m^{+} \rrbracket & \llbracket M^{+} \rrbracket+1
\end{array}\right)=\left(\begin{array}{cc}
m & M \\
\llbracket m \rrbracket & \llbracket M \rrbracket+1
\end{array}\right)\left(\begin{array}{cc}
1 & \delta \\
1-\delta & 1
\end{array}\right) .
$$

The above transformation of indices is bijective, since the determinant of the matrix is $1-\delta+\delta^{2}=$ 1 for each possible value of $\delta$. For Pythagorean scales, applying this recurrence we get again the values of Table B1. The iterations may start with $m=1, M=1, \llbracket m \rrbracket=\llbracket M \rrbracket=0, \delta=0$. It is possible to get a similar iterative process for the pair $(n, N)$,

$$
\left(\begin{array}{c}
n^{+} \\
N^{+}
\end{array}\right)=\left(\begin{array}{cc}
m & M \\
\llbracket m \rrbracket & \llbracket M \rrbracket+1
\end{array}\right)\left(\begin{array}{c}
1+\delta \\
2-\delta
\end{array}\right)=\left(\begin{array}{c}
n \\
N
\end{array}\right)+\left(\begin{array}{cc}
m & M \\
\llbracket m \rrbracket & \llbracket M \rrbracket+1
\end{array}\right)\left(\begin{array}{c}
\delta \\
1-\delta
\end{array}\right) .
$$

An equivalent algorithm ${ }^{17}$, although obtained from a different approach, is described in Noll (2007), in relation to the linear transformations providing addition of intervals in terms of a finitely generated free commutative group and to the intrinsic structure of the Stern-Brocot tree.

\footnotetext{
${ }^{17}$ According to equation (37), the subsequent matrices of indices are obtained multiplying by a matrix in one of the forms $L=\left(\begin{array}{ll}1 & 0 \\ 1 & 1\end{array}\right)$ or $R=\left(\begin{array}{ll}1 & 1 \\ 0 & 1\end{array}\right)$. These are equivalent to the $2 \times 2$ matrices representing the branches of the Stern-Brocot tree (Noll 2006, 2007; Jedrzejewski 2009, 2008), providing the strings of $L$ 's and $R$ 's that encode the subsequent nodes of the fractions $\frac{N}{n}$. This is equivalent to the retrograde Euclidean algorithm along the path to the root from that node in the Raney tree. On the other hand, the fractions $\frac{m}{M}$ follow a similar path in the Raney tree, with the same strings as $\frac{N}{n}$.
} 


\subsection{Condition for optimal scale}

While processing the iteration described in the past section, it is possible to determine the optimal scales.

THEOREM 5.1 The cyclic sequence next to $S_{n}^{h}$ changes the value of the digit $\delta$ if and only if $S_{n}^{h}$ is optimal.

Proof. Let us consider an optimal cyclic sequence $S_{n}^{h}$, i.e., $\left|\log \gamma_{n}\right|<\left|\log \gamma_{k}\right|, \forall k<n$, with extreme tones of indices $m, M$. There are two possible cases:

(1) If the last tone of $S_{n}^{h}$ is the maximum tone $\nu_{M}$, then $n=M>m$ and the comma of the optimal sequence is $\kappa_{n}=\gamma_{n}^{-1}>1$. Hence $\gamma_{n}<1$. For the next cyclic sequence $S_{n^{+}}^{h}$ with $n^{+}=m+M$, not necessarily optimal, since $S_{n}^{h}$ is optimal, owing to the first expression of equation (24) we have $1<\gamma_{m+M}<\frac{2}{\nu_{M}}<\nu_{m}$. Hence, $\gamma_{m+M}>1$.

Thus, if $S_{n}^{h}$ is optimal with $\gamma_{n}<1$ (therefore $\delta=1$ ), the next sequence $S_{n^{+}}^{h}$ has closure $\gamma_{n^{+}}>1$ (and $\delta=0$ ). If $S_{n}^{h}$ were not optimal, it would satisfy $1<\nu_{m}<\frac{2}{\nu_{M}}$, so that $\gamma_{m+M}=\frac{\nu_{m} \nu_{M}}{2}<1$ (and $\delta=1$ ), similarly to $\gamma_{n}$.

(2) If the last tone of $S_{n}^{h}$ is the minimum tone $\nu_{m}$, then $n=m>M$ and the comma of the optimal sequence is $\kappa_{n}=\gamma_{n}>1$. For the next cyclic sequence $S_{n^{+}}^{h}$ with $n^{+}=m+M$, not necessarily optimal, since $S_{n}^{h}$ is optimal, owing to the second expression of equation (24) we have $1<\frac{1}{\gamma_{m+M}}<\nu_{m}<\frac{2}{\nu_{M}}$. Hence, $\gamma_{m+M}<1$.

Thus, if $S_{n}^{h}$ is optimal with $\gamma_{n}>1$ (therefore $\delta=0$ ), the next sequence $S_{n^{+}}^{h}$ has closure $\gamma_{n^{+}}<1$ (and $\delta=1$ ). If $S_{n}^{h}$ were not optimal, it would satisfy $1<\frac{2}{\nu_{M}}<\nu_{m}$, so that $\gamma_{m+M}=\frac{\nu_{m} \nu_{M}}{2}>1(\delta=0)$, similarly to $\gamma_{n}$.

Thus, the way to determine optimal scales is as follows. If between two consecutive cyclic sequences, $S_{n}^{h}$ and $S_{n^{+}}^{h}, \delta$ changes, then the sequence $S_{n}^{h}$ and the scale $E_{n}^{h}$ are optimal. The extreme tones of this scale match those of the previous cyclic sequence $S_{n^{-}}^{h}$.

COROLlary 5.2 Consecutive optimal scales have alternate values of $\delta$ and the last iteration of their sequences closes alternatively at opposite extremes of the octave.

Such a behaviour for optimal scales is equivalent to the property of alternance of the continued fraction convergents remarked by Carey and Clampitt $(1989,2012)$, although here it has been obtained as a natural result of the process of building cyclic scales.

\section{Partition of the octave}

\subsection{Elementary factors}

From the classes of projection functions with indices in $\mathbb{Z}_{n}$, by taking into account their representatives in $\Omega_{0}$, we order by pitch the scale tones in [1,2). Let us bear in mind that the class of a power $\left(\bar{\pi}_{h}^{p}\right)^{q}$ has a representative given by $\pi_{h}^{\overline{p q}}$, where $\overline{p q}=p q \bmod n$. On the right side of $\nu_{0}=1$ the tones are generated by $n-1$ iterations of $\bar{\pi}_{h}^{m}$ as increasing pitches. Similarly, for the lower octave $\Omega_{-1}$, on the left side of 1 , we arrange them backward, generated by $n-1$ iterations of $\bar{\pi}_{h}^{M}$, as decreasing pitches (with their representatives reduced by a factor $\frac{1}{2}$ ). Then, the resulting tones remain ordered along two consecutive octaves as

$$
\cdots<\frac{1}{2} \pi_{h}^{\overline{m M}}<\cdots<\frac{1}{2} \pi_{h}^{\overline{2 M}}<\frac{1}{2} \pi_{h}^{\bar{M}}<1<\pi_{h}^{\bar{m}}<\pi_{h}^{\overline{2 m}}<\cdots<\pi_{h}^{\overline{M m}}<\cdots
$$


In particular, the interval between the extreme values displayed in equation (39) contains all the scale tones. It corresponds to the octave $\Omega_{0}^{\prime}=\left[\frac{1}{2} \pi_{h}^{\overline{m M}}, \pi_{h}^{\overline{m M}}\right)$. However, if in an attempt to write the scale tones in terms of the first $n$ iterates of equation (11), instead of equation (39) we had written the scale tones as

$$
\cdots<\left(\frac{\nu_{M}}{2}\right)^{m}<\cdots<\left(\frac{\nu_{M}}{2}\right)^{2}<\frac{\nu_{M}}{2}<1<\nu_{m}<\nu_{m}^{2}<\cdots<\nu_{m}^{M}<\cdots
$$

then, the above scales would not have coincided: while the former neglects the comma, the latter accumulates it. Although the frequencies in equation (40) belong to the FCs of the abstract scale $\bar{E}_{n}^{h}$, they do not necessarily match the scale tones of $E_{n}^{h}$ since they can differ in some commas. Nevertheless, we will prove that the interval $\Omega_{0}^{\prime}=\left[\left(\frac{\nu_{M}}{2}\right)^{m}, \nu_{m}^{M}\right)$ is exactly one octave.

\subsection{Fundamental relationship}

THEOREM 6.1 The frequency ratio of one octave is the product of $m$ factors $\frac{2}{\nu_{M}}$ and $M$ factors $\nu_{m}$, e.g.,

$$
\nu_{m}^{M}\left(\frac{2}{\nu_{M}}\right)^{m}=2 .
$$

Proof. It will be proved in several steps.

(a) By fixing $n \in \mathbb{R}$, for two values $x, y>0$ such that $y \neq \frac{1}{x}$, there is a single value $\alpha$ that satisfies the equation

$$
2 y^{n-\alpha}=x^{\alpha} .
$$

Indeed, by taking $\log _{2}$, we get $\alpha=\frac{1+n \log _{2} y}{\log _{2}(x y)}$, which is defined and is unique if $x y \neq 1$.

(b) If we add the conditions $y<1, x>1, \alpha>0$, and $n-\alpha>0$, then we are in the conditions of the problem we were considering (with $x=\nu_{m}, y=\frac{\nu_{M}}{2}$ ), so that the ratio between the extremes of the interval $\left[y^{n-\alpha}, x^{\alpha}\right)$ is 2 , corresponding to one octave. This will impose some conditions on $x$ and $y$.

Thus, we have $\log _{2} x>0, \alpha \log _{2} x>0$ and $\log _{2} y<0,(n-\alpha) \log _{2} y<0$. Therefore, the powers $y^{n-\alpha}<1$ and $x^{\alpha}>1$ remain on both sides of the fundamental tone, by fulfilling, owing to equation (42),

$$
2 y^{n-\alpha}<2 \Rightarrow x^{\alpha}<2 \Rightarrow x<2^{\frac{1}{\alpha}}, \quad x^{\alpha}>1 \Rightarrow 2 y^{n-\alpha}>1 \Rightarrow y>2^{\frac{1}{\alpha-n}} .
$$

(c) For $x=\nu_{m}$ and $y=\frac{\nu_{M}}{2}$, conditions (a) and (b) are satisfied. Since $n=M+m$, the value $\alpha=M$ fits $2\left(\frac{\nu_{M}}{2}\right)^{n-\alpha}=\nu_{m}^{\alpha}$. Therefore, it necessarily matches the one solution of equation (42).

By identifying the powers of 2 in both sides of equation (41), we derive the relationship that we shall refer to as the fundamental identity of $h$-cyclic scales:

COROLlaRY 6.2 The indices of the extreme tones satisfy the identity

$$
(\llbracket M \rrbracket+1) m-\llbracket m \rrbracket M=1 .
$$


We meet again Bézout's identity, $a m+b M=1$, with $m$ and $M$ coprime, but additionally we now have determined the values of the parameters $a$ and $b$. In addition, since $N=\llbracket m \rrbracket+\llbracket M \rrbracket+1$, we get straightforwardly ${ }^{18}$

$$
\begin{gathered}
N m-n \llbracket m \rrbracket=1 \\
n(\llbracket M \rrbracket+1)-N M=1
\end{gathered}
$$

To calculate the indices $m$ and $M$ one could apply the extended Euclidean algorithm to equation (45) if $N>n$, or to equation (46) if $n>N$, by starting from the values $n, N$ of the scale $E_{n}^{h}$. Appendix D details the algorithm.

The following boundaries are a straightforward consequence of equation (43).

Corollary 6.3 The extreme tones satisfy

$$
\frac{1}{2}<\left(\frac{\nu_{M}}{2}\right)^{m}, \quad \nu_{m}^{M}<2
$$

We denote the elementary factors (corresponding to elementary intervals in $S_{0}$ ) by $D \equiv \frac{2}{\nu_{M}}$, $U \equiv \nu_{m}$. By equation (18), we have $\gamma_{n}=\frac{U}{D}$, so that, in the frequency space, the factors $U$ and $D$ compose the spectrum of the interval that spans one step.

Although in the current paper we will not discuss the exact order of these factors between the scale tones in the octave $\Omega_{0}$, we provide an easy way to find it. It suffices to solve the system resulting from the equation $\nu_{k}=\nu_{m}^{r}\left(\frac{2}{\nu_{M}}\right)^{s}$ for $0 \leq k<n, 0 \leq r<M, 0 \leq s<m$; that is,

$$
\left(\begin{array}{c}
k \\
\llbracket k \rrbracket
\end{array}\right)=\left(\begin{array}{cc}
m & -M \\
\llbracket m \rrbracket & -(\llbracket M \rrbracket+1)
\end{array}\right)\left(\begin{array}{c}
r \\
s
\end{array}\right) .
$$

According to equation (44), the system determinant is non-zero; hence, there exists an isomorphism between both pairs of indices describing the scale. Therefore, each iteration $\nu_{k}$ has a unique expression in terms of the elementary factors $U$ and $D$. These frequency ratios (with value greater than 1) play the roles of generator/co-generator ${ }^{19}$. Since within the octave there are $n$ scale tones and $n$ elementary factors, we conclude:

COROLlary 6.4 The factors $U$ and $D$ are the only possible ratios between tones, which generate the scale tones and introduce the non-equal temperament.

\subsection{Partition of $j$ octaves}

The previous results can be generalized.

THEOREM 6.5 The frequency ratios $\nu_{l}$ and $\frac{2}{\nu_{L}}$, with $l \in\{1, \ldots, n-1\}$ and $L=n-l$, compose the spectrum of the specific interval that spans a number of steps $j=(\llbracket L \rrbracket+1) l-\llbracket l \rrbracket L$.

\footnotetext{
${ }^{18}$ One consequence of the fundamental identity is that all the following pairs are coprime: $(m, M) ;(\llbracket M \rrbracket+1, M) ;(\llbracket M \rrbracket+1, \llbracket m \rrbracket) ;(m, \llbracket m \rrbracket) ;(m, n) ;(M, n) ;(n, N) ;(\llbracket m \rrbracket, N) ;(\llbracket M \rrbracket+1, N)$.

${ }^{19}$ The procedure to determine cyclic scales and their refinements is closely related to the concept of mechanical or Sturmian words used in the new approaches to the theory of well-formed scales and modes (Noll 2008; Clampitt and Noll 2011; Noll 2015) based on methods of combinatorics on words (e.g., Lothaire 2002). A cyclic scale can be defined as a Christoffel word of the alphabet $\{U, D\}$ with slope $\frac{m}{M}$ and length $n$. For cyclic scales, the first step of the octave after 1 must be $U$, and the last step before 2 must be $D$, although generic scales do not need to satisfy such a requirement. Given a scale whose factors satisfy $U^{M} D^{m}=2$, if $U<D$ then, owing to Myhill's property, it can be refined by factorizing $D=U D^{\prime}$, otherwise by factorizing $U=U^{\prime} D$, and so on. The refinement of cyclic scales correspond to the binary tree of Christoffel words.
} 
Proof. We use the more general relationship

$$
2^{j} y^{n-\alpha}=x^{\alpha}
$$

for $j \geq 1$, with the same reasoning and under the same conditions as in the above section. Then, the values $\alpha$ and $j$ are linked by the equation $\alpha=\frac{j+n \log _{2} y}{\log _{2}(x y)}$. By taking $x=\nu_{l}$ and $y=\frac{\nu_{L}}{2}$, i.e., $L=\alpha$ and $l=n-\alpha$, we then obtain

$$
\nu_{l}^{L}\left(\frac{2}{\nu_{L}}\right)^{l}=2^{j} ; \quad l, L \in\{1, \ldots, n-1\}
$$

that generalizes Theorem 6.1. It is easy to see that by interchanging $l$ and $L$ the power of 2 becomes $n-j$. The foregoing equation explains how each pair of pseudo-complementary tones induces a partition in a specific set of octaves.

By identifying the powers of 2 in both sides of the above equation we get the identity

$$
(\llbracket L \rrbracket+1) l-\llbracket l \rrbracket L=j
$$

for values $j \in\{1, \ldots, n-1\}$, that generalizes Corollary 6.2 .

Similarly, the generalization of Corollary 6.3 leads to the following conditions

$$
\frac{1}{2^{j}}<\left(\frac{\nu_{L}}{2}\right)^{l}, \quad \nu_{l}^{L}<2^{j} .
$$

By taking into account equation (35) and writing $L=n-l$, equation (51) can be rewritten depending on $n$ and $N$. Since $(\llbracket(n-l) \rrbracket+1) l-\llbracket l \rrbracket(n-l)=(\llbracket(n-l) \rrbracket+\llbracket l \rrbracket+1) l-\llbracket l \rrbracket n$, we get

$$
N l-\llbracket l \rrbracket n=j .
$$

In particular, for the first iterate $l=1$, we get $j=N$ since $\llbracket 1 \rrbracket=0$.

COROLLARY 6.6 The deviation from equal temperament is given as $\nu_{l}=2^{\frac{j}{n}} \gamma_{n}^{\frac{l}{n}}$ and $\frac{2}{\nu_{L}}=2^{\frac{j}{n}} \gamma_{n}^{-\frac{L}{n}}$.

Proof. As defined in Section 4.2, $\nu_{l}$ and $\nu_{L}$ are pseudo-complementary. Thus, by equation (32) we have $\nu_{l} \nu_{L}=2 \gamma_{n}$, therefore $x y=\gamma_{n}$. Hence, in equation (49), we get

$$
2^{j} y^{n}=\gamma_{n}^{L} \Longrightarrow y=2^{-\frac{j}{n}} \gamma_{n}^{\frac{L}{n}}
$$

Therefore,

$$
\frac{2}{\nu_{L}}=\frac{1}{y}=2^{\frac{j}{n}} \gamma_{n}^{-\frac{L}{n}}, \quad \nu_{l}=x=\frac{\gamma_{n}}{y}=2^{\frac{j}{n}} \gamma_{n}^{\frac{l}{n}}
$$

When $\gamma_{n} \approx 1$, we get the approximations $\nu_{l} \approx 2^{\frac{j}{n}}, \nu_{L} \approx 2^{\frac{n-j}{n}}$, which determines what couple of indices $l$ and $L$ produce a particular value of $j$ in equation (50). The set of indices can be extended to 0 to include the fundamental tone. Hence, $j$ is the chromatic cardinality of $\nu_{l}$.

\subsection{Best equal temperament scales}

These results can be used to link the $n$-tone cyclic scale to the corresponding $n$-TET scale. 
Equation (54) gives the exact deviation of the tones of a non-degenerated cyclic scale from an equal temperament scale in terms of the scale closure. These expressions can be used in order to study the proximity of these scales (e.g., Douthett and Krantz 2007; Krantz and Douthett 2011; Liern 2015). As previously noticed, a good approximation between the $\mathrm{FC} h$ of the generator interval of the cyclic scale and the corresponding tone $2^{\frac{N}{n}}$ of the $n$-TET scale is not a sufficient condition for a good approximation between the other tones.

By measuring in cents the interval between $\nu_{l}$ and $2^{\frac{j}{n}}$ for $l \in\{1, \ldots, n-1\}$, bearing in mind equation (20), we get $1200\left|\log _{2}\left(\nu_{l} / 2^{\frac{j}{n}}\right)\right|=\frac{l}{n} \phi\left(\kappa_{n}\right)$. In order to associate unambiguously each note of the $h$-cyclic scale with the corresponding one of the $n$-TET scale, it is necessary for those intervals, in particular the wider one corresponding to $l=n-1$, to be smaller than half an elementary interval of the $n$-TET scale, i.e., $\frac{1200}{2 n}$. Therefore, to get a $n$-TET scale that unambiguously approximates all the notes of a $h$-cyclic scale we should add the condition

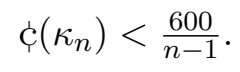

Then we get a sufficient condition for a good approximation between both scales, similar to the sufficient conditions given by Liern (2015) in order to compare different types of tuning systems. This allows to distinguish, among the good approximations of $\log _{2} h$, what $n$-TET scales provide optimal fittings of $h$-cyclic scales. For instance, for $n=12$ the values $\dot{c}\left(\kappa_{n}\right)=23.5$ and $\frac{600}{n-1}=54.5$ guarantee a good fitting, for $n=41$ the values $\phi\left(\kappa_{n}\right)=20$ and $\frac{600}{n-1}=15$ indicate that some note is not fitting well.

\subsection{Generator and co-generator pairs}

Equation (A2) can also be written in terms of coprime indices $l$ and $L=n-l$, as

$$
\pi_{h}^{k}=\pi_{h}^{l r} \pi_{h}^{-L r} \Longleftrightarrow k=l r-L s ; \quad 0 \leq k<n, 0 \leq r<L, 0 \leq s<l
$$

so that the scale tones can be expressed as $\nu_{k}=\nu_{l}^{r}\left(\frac{2}{\nu_{L}}\right)^{s}$. Each generator has its pseudocomplementary co-generator. Among the indices coprime with $n$ we have found several possible values, namely, $l=1$ is the index of the generator $h$ giving the tones ordered according to its iterates, $l=m$ generates the tones increasingly from the minimum tone, $l=M$ provides the tones ordered decreasingly from the maximum tone, and the chromatic length of the generator $l=N$, if it does not match any of the previous ones, also generates the scale in a different order.

\section{Conclusions}

In the current paper a new approach to generalized Pythagorean scales, developed in the specific level of frequency classes, has been described. The abstract scale is formalized in terms of a cyclic group of classes of projection functions, more similar to Hellegouarch's (1999) factor groups than to Carey and Clampitt's (1989) method of continued fractions. Their representatives are used to built cyclic sequences of tone iterates satisfying the closure condition. The refinement of cyclic sequences with regard to the best closure provides a chain of progressively finer cyclic sequences leading to a generating algorithm for cyclic scales. The optimal closure of a $n$-tone cyclic sequence is tantamount to obtaining the best comma and, therefore, the best rational approximation $\frac{N}{n}$ of $\log _{2} h$. Optimal scales provide the best approximations from both sides, while non-optimal scales converge from one side. On the other hand, good rational approximations of $\log _{2} h$ are associated with $n$-TET scales providing the best estimations of the generator FC.

The algorithm is based on the following properties, providing a new strategy to prove some basic properties of well-formed scales. If $S_{n^{-}}^{h}$ is a sequence, not necessarily cyclic, and has extreme 
tones of indices $m, M$, then the sequence $S_{n}^{h}$ with $n=m+M$ improves at least one of the marginal ratios of $S_{n^{-}}^{h}$ and is cyclic. Furthermore, none of the marginal ratios of a cyclic sequence $S_{n^{-}}^{h}$ can be improved with a sequence of an order lower than $n=M+m$. Therefore, it is possible to build a chain $\ldots \subset S_{n^{-}}^{h} \subset S_{n}^{h} \subset \ldots$ of progressively finer cyclic sequences with $n=m+M$. $S_{n}^{h}$ generates the scale $E_{n}^{h}$, which also has indices of the extreme tones $(m, M)$.

The algorithm provides itself several parameters which characterize a cyclic scale. For a $n$-tone scale, the main parameter is the scale closure $\gamma_{n}=U / D$, quotient of the two elementary factors $U=\nu_{m}$ and $D=\frac{2}{\nu_{M}}$ generating the scale tones. It accounts for the equal spectrum variations of the scale, that can be expressed in the frequency domain as $\gamma_{n}=\frac{\nu_{m} \nu_{M}}{2}=\frac{h^{n}}{2^{N}}$, either depending on the indices of the extreme tones (the generic widths of the step intervals) or the chromatic length of the generator and the chromatic cardinality of the scale. In addition, the scale closure can be computed as $\gamma_{n}=\frac{\nu_{n-k} \nu_{k}}{2}$ for $k=1, \ldots, n-1$, being determined from pairs of factors $\nu_{k}$ and $\frac{2}{\nu_{n-k}}$ (associated with pseudo-complementary tones) composing the different step spectra in relation to Myhill's property.

The chromatic length $N$ and the index of octave $\llbracket n \rrbracket$ are significant integers. The former can be computed as $N=\llbracket n-k \rrbracket+\llbracket k \rrbracket+1 ; k=1, \ldots, n-1$; and their difference, the scale digit $\delta$, takes value 0 or 1 depending on whether $\gamma_{n}$ is greater or smaller than 1 . Therefore, we can know whether the $n$-th iteration closes near 1 or 2 directly from the scale digit. On the other hand, $\delta$ relates the indices of the extremes tones of a cyclic scale $E_{n}^{h}$ to similar values of the next and previous cyclic scales of the chain according to equation (37), leading to an alternative matrix algorithm. This reproduces the structure of the Stern-Bocot tree, as in Noll (2007), although here it is rooted on a less abstract approach. For the chain of finer cyclic sequences, according to the matrix algorithm, it has been proved that the next cyclic sequence to $S_{n}^{h}$ changes the value of $\delta$ if and only if $S_{n}^{h}$ is optimal. Hence, there are two results to point out. One is new: optimal scales can be determined from oscillations in $\delta$. The other already known: consecutive optimal scales close their cycle of tone iterates at opposite extremes of the octave.

An ad hoc approach is proposed to prove that the whole octave of a non-degenerate cyclic scale remains composed of $n$ elementary intervals separating the scale notes, from which $m$ correspond to factor $D$, and $M$ to factor $U$. This result leads to an explicit Bézout's identity relating the indices of the extreme tones in either of its forms, equations (44), (45), or (46). From the latter, given the pair $(n, N)$, the indices of the extremes tones $(m, M)$ can be determined by applying the extended Euclidean algorithm, which is equivalent to determining the previous cyclic scale in the chain. From there, a new and more general relationship is derived. For each index $l \in\{0, \ldots, n-1\}$ and $L=n-l$, not necessarily coprime with $n$, the step-interval $j$ corresponding to the spectrum of frequency ratios $\left(\nu_{l}, \frac{2}{\nu_{L}}\right)$ is determined such that each pair of frequency ratios induces an exact partition of $j$ octaves. Therefore, $j$ is the chromatic cardinality corresponding to the scale tone $\nu_{l}$. If the indices $l, n$ are coprime, the above frequency ratios are generator and co-generator of the scale. For each value $j=0, \ldots, n-1$, if $\gamma_{n} \rightarrow 1$, the tones $\nu_{l}$ and $\nu_{L}$ converge to complementary tones of a $n$-TET scale, whose quality of approximation to the cyclic scale has been explicitly obtained.

\section{Appendix A. Bézout's identity}

Instead of using classes of projection functions with indices in $\mathbb{Z}_{n}$ we may work with projection functions over $\mathbb{Z}$ according to the rules of modular arithmetic, by applying congruences modulo $n$ over the indices. Since two projection functions $\pi_{h}^{p}, \pi_{h}^{q}$ belong to the same class if there is a number $k \in \mathbb{Z}$ such that $\pi_{h}^{p}=\pi_{h}^{q} \pi_{h}^{k n}$, then $p-q$ is a multiple of $n$. Therefore, $\pi_{h}^{p}$ and $\pi_{h}^{q}$ are equivalent projection functions if and only if $p \equiv q(\bmod n)$.

Assume $p, n \in \mathbb{Z}$, with $p<n$. Bézout's lemma states that $p$ and $n$ are coprime if and only if there are two integers $a$ and $b$ that satisfy $1=p a-n b$. This equation admits infinitely many 
pairs of solutions, although if $p$ and $n$ are positive, there exists a unique couple of values $a, b$ satisfying $0 \leq a<n, 0 \leq b<p$. The three pairs $a$ and $b, a$ and $n, p$ and $b$ are couples of coprime numbers. If we multiply the above equation by an integer $k$ such that $0<k<n$, and write $x=k a, y=k b$, we get the Diophantine equation

$$
k=p x-n y .
$$

For positive integers $p, n$ there exists only one pair of values $x, y$ satisfying $0 \leq x<n, 0 \leq y<p$. The equation (A1) is also valid for the value $k=0$ in the trivial case $x=y=0$. Hence, the projection functions $\left\{\pi_{h}^{k}, 0 \leq k<n\right\}$ providing the tones of the scale can be written as

$$
\pi_{h}^{k}=\pi_{h}^{p x} \pi_{h}^{-n y}
$$

with $p<n$, coprime. So, any projection function $\pi_{h}^{k}$, with $0 \leq k<n$, is of the same class that $\pi_{h^{p}}^{x}$ for a single value $0 \leq x<n$ satisfying equation (A1).

\section{Appendix B. Generating algorithm}

We are dealing with cyclic sequences $S_{n}^{h}$, so that $\nu_{n}$ is always an extreme tone. The starting sequence is $S_{2}^{h}$ with two tones $\nu_{1}, \nu_{2}$. The indices of the extreme tones are initialized as $m=$ $1+I, M=2-I$, where $I$ will take the value 0 or 1 depending on the value $h$. For instance, if $h=3$ then $I=1$, since the extreme tones have indices $m=2$ and $M=1$. These values provide marginal ratios that are compared with the absolute ratio of the unit sequence $S_{1}^{h}$. In general, if the sequence previous to $S_{n}^{h}$ has absolute ratio $\rho_{0}$, we need to compute the marginal ratios of the extreme tones $m$ and $M$ of the sequence $S_{n}^{h}$ and evaluate the minimum marginal ratio $\rho$. If $\rho<\rho_{0}$, the minimum marginal ratio has decreased and then $S_{n}^{h}$ is an optimal sequence with comma $\kappa_{n}=\rho$, leading to an optimal scale $E_{n}^{h}$. However, if $\rho=\rho_{0}$, the marginal ratio remains equal to the previous sequence and, therefore, the refined sequence is not optimal, although it is still cyclic. In the next iteration, the refined sequence with $m+M$ notes is considered, with one of the extreme tones improved with regard to the previous sequence. This new extreme tone will be the minimum tone if $\frac{\nu_{M} \nu_{m}}{2}>1$, and the maximum otherwise. Then the procedure is repeated, by comparing this sequence to the previous one to see whether the absolute ratio is improved.

As an example, cyclic scales $E_{n}^{h}$ for $h=3$ and 5 are calculated. For $h=3$, Table B1 lists non-optimal Pythagorean scales on gray background and optimal on white, by displaying the cardinal of the scale, the indices of the extreme tones $m, M$, besides other quantities. Other values of $n$, such as $n=7$ and 29, correspond to good approximations (marked with asterisk) of $\log _{2} 3$. They provide non-optimal Pythagorean scales related to $n$-TET scales providing an optimal approximation of the class of the generator tone. For instance, 29 is the lowest number of equal divisions of the octave that produces a better perfect fifth than the 12-TET scale. There are also non-optimal scales providing equal temperament scales that are not good approximations of $\log _{2} 3$, such as $n=17$. For $h=5$, Table B2 displays the first 5-cyclic scales. In such a case the mediant is prioritized in front of the dominant. Here, optimal scales corresponds to the best rational approximations of $\log _{2} 5$.

\section{Appendix C. Best and good rational approximations}

We review the concept of rational approximation of a real number (e.g., Loya 2017). A fraction $\frac{\alpha}{\beta}$ with $\alpha, \beta>0$ is a good rational approximation of a real number $r>0$ if any rational $\frac{a}{b}$ with 


\begin{tabular}{|c|c|c|c|c|c|c|}
\hline$n$ & $m$ & $M$ & $N$ & $\delta$ & $\varepsilon_{n}$ & 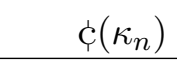 \\
\hline $2^{*}$ & 1 & 1 & 3 & 0 & $8.50 \mathrm{E}-02$ & $2.04 \mathrm{E}+02$ \\
\hline $3^{*}$ & 2 & 1 & 5 & 1 & $-8.17 \mathrm{E}-02$ & $2.94 \mathrm{E}+02$ \\
\hline $5^{*}$ & 2 & 3 & 8 & 1 & $-1.50 \mathrm{E}-02$ & $9.02 \mathrm{E}+01$ \\
\hline $7^{*}$ & 2 & 5 & 11 & 0 & $1.35 \mathrm{E}-02$ & $1.14 \mathrm{E}+02$ \\
\hline $12^{*}$ & 7 & 5 & 19 & 0 & 1.63E-03 & $2.35 \mathrm{E}+01$ \\
\hline 17 & 12 & 5 & 27 & 1 & $-3.27 \mathrm{E}-03$ & $6.68 \mathrm{E}+01$ \\
\hline $29^{*}$ & 12 & 17 & 46 & 1 & $-1.24 \mathrm{E}-03$ & $4.33 \mathrm{E}+01$ \\
\hline $41^{*}$ & 12 & 29 & 65 & 1 & $-4.03 \mathrm{E}-04$ & $1.98 \mathrm{E}+01$ \\
\hline $53^{*}$ & 12 & 41 & 84 & 0 & $5.68 \mathrm{E}-05$ & $3.62 \mathrm{E}+00$ \\
\hline 94 & 53 & 41 & 149 & 1 & $-1.44 \mathrm{E}-04$ & $1.62 \mathrm{E}+01$ \\
\hline 147 & 53 & 94 & 233 & 1 & $-7.15 \mathrm{E}-05$ & $1.26 \mathrm{E}+01$ \\
\hline $200^{*}$ & 53 & 147 & 317 & 1 & $-3.75 \mathrm{E}-05$ & $9.00 \mathrm{E}+00$ \\
\hline $253^{*}$ & 53 & 200 & 401 & 1 & $-1.77 \mathrm{E}-05$ & $5.38 \mathrm{E}+00$ \\
\hline $306^{*}$ & 53 & 253 & 485 & 1 & $-4.82 \mathrm{E}-06$ & $1.77 \mathrm{E}+00$ \\
\hline $359^{*}$ & 53 & 306 & 569 & 0 & $4.28 \mathrm{E}-06$ & $1.85 \mathrm{E}+00$ \\
\hline $665^{*}$ & 359 & 306 & 1054 & 0 & $9.47 \mathrm{E}-08$ & $7.56 \mathrm{E}-02$ \\
\hline 971 & 665 & 306 & 1539 & 1 & $-1.45 \mathrm{E}-06$ & $1.69 \mathrm{E}+00$ \\
\hline 1636 & 665 & 971 & 2593 & 1 & $-8.24 \mathrm{E}-07$ & $1.62 \mathrm{E}+00$ \\
\hline 2301 & 665 & 1636 & 3647 & 1 & $-5.59 \mathrm{E}-07$ & $1.54 \mathrm{E}+00$ \\
\hline 2966 & 665 & 2301 & 4701 & 1 & $-4.12 \mathrm{E}-07$ & $1.47 \mathrm{E}+00$ \\
\hline 3631 & 665 & 2966 & 5755 & 1 & $-3.19 \mathrm{E}-07$ & $1.39 \mathrm{E}+00$ \\
\hline 4296 & 665 & 3631 & 6809 & 1 & $-2.55 \mathrm{E}-07$ & $1.32 \mathrm{E}+00$ \\
\hline 4961 & 665 & 4296 & 7863 & 1 & $-2.08 \mathrm{E}-07$ & $1.24 \mathrm{E}+00$ \\
\hline 5626 & 665 & 4961 & 8917 & 1 & $-1.73 \mathrm{E}-07$ & $1.17 \mathrm{E}+00$ \\
\hline 6291 & 665 & 5626 & 9971 & 1 & $-1.44 \mathrm{E}-07$ & $1.09 \mathrm{E}+00$ \\
\hline 6956 & 665 & 6291 & 11025 & 1 & $-1.21 \mathrm{E}-07$ & $1.01 \mathrm{E}+00$ \\
\hline 7621 & 665 & 6956 & 12079 & 1 & $-1.03 \mathrm{E}-07$ & $9.38 \mathrm{E}-01$ \\
\hline $8286^{*}$ & 665 & 7621 & 13133 & 1 & $-8.68 \mathrm{E}-08$ & 8.63E-01 \\
\hline $8951^{*}$ & 665 & 8286 & 14187 & 1 & $-7.33 \mathrm{E}-08$ & 7.87E-01 \\
\hline $9616^{*}$ & 665 & 8951 & 15241 & 1 & $-6.17 \mathrm{E}-08$ & $7.12 \mathrm{E}-01$ \\
\hline $10281^{*}$ & 665 & 9616 & 16295 & 1 & $-5.16 \mathrm{E}-08$ & $6.36 \mathrm{E}-01$ \\
\hline $10946^{*}$ & 665 & 10281 & 17349 & 1 & $-4.27 \mathrm{E}-08$ & $5.61 \mathrm{E}-01$ \\
\hline $11611^{*}$ & 665 & 10946 & 18403 & 1 & $-3.48 \mathrm{E}-08$ & 4.85E-01 \\
\hline $12276^{*}$ & 665 & 11611 & 19457 & 1 & $-2.78 \mathrm{E}-08$ & $4.09 \mathrm{E}-01$ \\
\hline $12941^{*}$ & 665 & 12276 & 20511 & 1 & $-2.15 \mathrm{E}-08$ & $3.34 \mathrm{E}-01$ \\
\hline $13606^{*}$ & 665 & 12941 & 21565 & 1 & $-1.58 \mathrm{E}-08$ & $2.58 \mathrm{E}-01$ \\
\hline $14271^{*}$ & 665 & 13606 & 22619 & 1 & $-1.07 \mathrm{E}-08$ & $1.83 \mathrm{E}-01$ \\
\hline $14936^{*}$ & 665 & 14271 & 23673 & 1 & $-5.97 \mathrm{E}-09$ & $1.07 \mathrm{E}-01$ \\
\hline $15601^{*}$ & 665 & 14936 & 24727 & 1 & $-1.68 \mathrm{E}-09$ & $3.15 \mathrm{E}-02$ \\
\hline 16266 & 665 & 15601 & 25781 & 0 & $2.26 \mathrm{E}-09$ & $4.41 \mathrm{E}-02$ \\
\hline $31867^{*}$ & 16266 & 15601 & 50508 & 0 & $3.29 \mathrm{E}-10$ & $1.26 \mathrm{E}-02$ \\
\hline 47468 & 31867 & 15601 & 75235 & 1 & $-3.32 \mathrm{E}-10$ & $1.89 \mathrm{E}-02$ \\
\hline $79335^{*}$ & 31867 & 47468 & 125743 & 1 & $-6.66 \mathrm{E}-11$ & $6.34 \mathrm{E}-03$ \\
\hline $111202^{*}$ & 31867 & 79335 & 176251 & 0 & $4.67 \mathrm{E}-11$ & $6.23 \mathrm{E}-03$ \\
\hline
\end{tabular}

Table B1. First $n$-tone Pythagorean scales $(h=3)$. Optimal Pythagorean scales, corresponding to the best rational approximations of $\log _{2} 3$, are listed on white background, non-optimal scales on gray. Good approximations are marked with asterisk. The comma $\kappa_{n}$ is measured in cents and $\varepsilon_{n}=\log _{2} 3-\frac{N}{n}$. 


\begin{tabular}{|c|c|c|c|c|c|c|}
\hline$n$ & $m$ & $M$ & $N$ & $\delta$ & $\varepsilon_{n}$ & 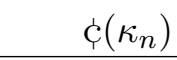 \\
\hline $2^{*}$ & $\overline{1}$ & $\overline{1}$ & $\overline{5}$ & $\bar{~} 1$ & 1.78E-01 & $\bar{c} 4.27 \mathrm{E}+02$ \\
\hline $3^{*}$ & 1 & 2 & 7 & 1 & $1.14 \mathrm{E}-02$ & $4.11 \mathrm{E}+01$ \\
\hline 4 & 1 & 3 & 9 & 0 & $-7.19 \mathrm{E}-02$ & $3.45 \mathrm{E}+02$ \\
\hline 7 & 4 & 3 & 16 & 0 & $-3.62 \mathrm{E}-02$ & $3.04 \mathrm{E}+02$ \\
\hline 10 & 7 & 3 & 23 & 0 & $-2.19 \mathrm{E}-02$ & $2.63 \mathrm{E}+02$ \\
\hline 13 & 10 & 3 & 30 & 0 & $-1.42 \mathrm{E}-02$ & $2.22 \mathrm{E}+02$ \\
\hline $16^{*}$ & 13 & 3 & 37 & 0 & $-9.43 \mathrm{E}-03$ & $1.81 \mathrm{E}+02$ \\
\hline $19^{*}$ & 16 & 3 & 44 & 0 & $-6.14 \mathrm{E}-03$ & $1.40 \mathrm{E}+02$ \\
\hline $22^{*}$ & 19 & 3 & 51 & 0 & $-3.75 \mathrm{E}-03$ & $9.89 \mathrm{E}+01$ \\
\hline $25^{*}$ & 22 & 3 & 58 & 0 & $-1.93 \mathrm{E}-03$ & $5.78 \mathrm{E}+01$ \\
\hline $28^{*}$ & 25 & 3 & 65 & 0 & $-5.00 \mathrm{E}-04$ & $1.68 \mathrm{E}+01$ \\
\hline 31 & 2 & 3 & 7 & & $6.53 \mathrm{E}-04$ & $2.43 \mathrm{E}+01$ \\
\hline $59^{*}$ & 2 & 31 & & & $1.06 \mathrm{E}-04$ & $7.49 \mathrm{E}+00$ \\
\hline $87^{*}$ & 28 & 59 & 02 & 0 & $-8.90 \mathrm{E}-05$ & $9.29 \mathrm{E}+00$ \\
\hline $146^{*}$ & 87 & 59 & 339 & 0 & $-1.03 \mathrm{E}-05$ & $1.80 \mathrm{E}+00$ \\
\hline 205 & & 59 & & & $2.31 \mathrm{E}-05$ & $5.69 \mathrm{E}+00$ \\
\hline $351^{*}$ & & 205 & 5 & & $9.23 \mathrm{E}-06$ & $3.89 \mathrm{E}+00$ \\
\hline $497^{*}$ & & 351 & 54 & 1 & $3.49 \mathrm{E}-06$ & $2.08 \mathrm{E}+00$ \\
\hline $643^{*}$ & 146 & 497 & 493 & 1 & 3.65E-07 & 2.82E-01 \\
\hline 789 & 146 & 643 & & 0 & $-1.61 \mathrm{E}-06$ & $1.52 \mathrm{E}+00$ \\
\hline 1432 & 789 & 643 & & 0 & $-7.21 \mathrm{E}-07$ & $1.24 \mathrm{E}+00$ \\
\hline 2075 & 1432 & 643 & 4818 & 0 & $-3.84 \mathrm{E}-07$ & $9.56 \mathrm{E}-01$ \\
\hline $2718^{*}$ & 2075 & 643 & & 0 & $-2.07 \mathrm{E}-07$ & $6.74 \mathrm{E}-01$ \\
\hline $3361^{*}$ & 2718 & 643 & & 0 & $-9.73 \mathrm{E}-08$ & $3.92 \mathrm{E}-01$ \\
\hline 400 & & & & 0 & $-2.30 \mathrm{E}-08$ & $1.10 \mathrm{E}-01$ \\
\hline 464 & & & & & -08 & $1.72 \mathrm{E}-01$ \\
\hline 865 & & & & 1 & & $6.14 \mathrm{E}-02$ \\
\hline 1265 & & & & 0 & $-3.22 \mathrm{E}-09$ & $4.90 \mathrm{E}-02$ \\
\hline $21306^{*}$ & 12655 & 8651 & 49471 & 1 & $4.85 \mathrm{E}-10$ & $1.24 \mathrm{E}-02$ \\
\hline 33961 & 12655 & 21306 & 78855 & 0 & $-8.97 \mathrm{E}-10$ & $3.66 \mathrm{E}-02$ \\
\hline $55267^{*}$ & 33961 & 21306 & 128326 & 0 & $-3.64 \mathrm{E}-10$ & $2.42 \mathrm{E}-02$ \\
\hline 76573 & 55267 & & 177797 & 0 & $-1.28 \mathrm{E}-10$ & $1.18 \mathrm{E}-02$ \\
\hline 97879* & 76573 & 21306 & 227268 & 1 & $5.31 \mathrm{E}-12$ & $6.24 \mathrm{E}-04$ \\
\hline
\end{tabular}

Table B2. Firsts $n$-tone 5 -cyclic scales. Optimal scales, corresponding to the best rational approximations of $\log _{2} 5$, are listed on white background, non-optimal scales on gray. Good approximations are marked with asterisk. The comma $\kappa_{n}$ is measured in cents and $\varepsilon_{n}=\log _{2} 5-\frac{N}{n}$.

$a, b>0$ such that $\frac{a}{b} \neq \frac{\alpha}{\beta}$ and $1 \leq b \leq \beta$ satisfies

$$
\left|r-\frac{\alpha}{\beta}\right|<\left|r-\frac{a}{b}\right|
$$

This inequality is equivalent to $|\beta r-\alpha|<\frac{\beta}{b}|b r-a|$. Since $\frac{\beta}{b} \geq 1$, it is possible to require a stronger condition. A fraction $\frac{\alpha}{\beta}$ satisfying

$$
|\beta r-\alpha|<|b r-a|
$$

is called a best approximation of $r$. 


\begin{tabular}{c||cc|ccccc}
$i$ & 0 & 1 & 2 & 3 & 4 & 5 & 6 \\
\hline$d_{i}$ & 53 & 31 & 22 & 9 & 4 & 1 & 0 \\
$q_{i}$ & & 1 & 1 & 2 & 2 & 4 & \\
\hline$x_{i}$ & 1 & 0 & 1 & -1 & 3 & -7 & \\
$y_{i}$ & 0 & 1 & -1 & 2 & -5 & 12 &
\end{tabular}

Table D1. Iterations of the extended Euclidean algorithm for $n=53, N=31$.

The Best Approximation Theorem states that every best approximation of a real number is a convergent of its canonic continued fraction expansion and, conversely, each of the convergents is a best approximation. This guarantees that the best approximations of a real number are provided by the convergents of its canonical continued fractions. Thus, equation (C2) will yield the continued fraction full convergents without having to calculate them explicitly. In addition, with regard to one-sided approximations, we point out two facts. On the one hand, every onesided good approximation $\frac{\alpha}{\beta}$, i.e., satisisfying $0<r-\frac{\alpha}{\beta}<r-\frac{a}{b}$ or $r-\frac{a}{b}<r-\frac{\alpha}{\beta}<0$, provides a convergent or a semi-convergent (intermediate fraction) of $r$. On the other hand, every convergent and semi-convergent of $r$ is a one-sided best approximation, i.e., satisfies $0<\beta r-\alpha<b r-a$ or $b r-a<\beta r-\alpha<0$. An interesting geometrical interpretation of convergents and semi-convergents with regard to well formed scales is given in Hall and Josić (2001).

\section{Appendix D. Extreme tones}

The Euclidean algorithm allows to find the greatest common divisor of two integers $a>b>0$ from a succession of remainders of Euclidean divisions, so that the last non-zero remainder is $d=\operatorname{gcd}(a, b)$. Starting from $d_{0}=a, d_{1}=b$, the Euclidean division $\left\lfloor d_{0} / d_{1}\right\rfloor$ provides the quotient $q_{1}$ and the remainder $r_{1}$, satisfying $r_{1}=d_{0}-q_{1} d_{1}$. Hence, the values

$$
d_{i+1} \equiv r_{i}=d_{i-1}-q_{i} d_{i}, \quad i \geq 1
$$

form a decreasing sequence so that if $d_{k+1}=0$ then $d_{k}=d$. The extended Euclidean algorithm is an improvement of the previous one that, in addition to calculating $d$, provides the two pairs of integers $x$ and $y$ satisfying Bézout's identity $d=a x+b y ;|x|<|b|,|y|<|a|$.

If $x, y$ are solutions and $x<0$, then $x+b>0$ and $y-a$ are solutions too, and if $y<0$, then $x-b$ and $y+a>0$ also are. The way to proceed is to define the variables ${ }^{20}$

$$
x_{0}=y_{1}=1, x_{1}=y_{0}=0 ; \quad x_{i+1}=x_{i-1}-q_{i} x_{i}, \quad y_{i+1}=y_{i-1}-q_{i} y_{i}, i \geq 1
$$

satisfying $d_{i}=a x_{i}+b y_{i}, i<k$. For $i=k$, we get the coefficients of Bézout's identity $d=a x+b y$.

Therefore, if $n>N$, this method can be applied to equation (46) with $a=n, b=N$, $x=\llbracket M \rrbracket+1$, and $y=-M$, till reaching $d=1$. In Table D1 an example shows how to determine the minimum tone of the Pythagorean scale $\left(h=\frac{3}{2}\right)$ with $n=53$ tones and $N=31$. The rows $d_{i}, q_{i}$ follow the conventional Euclidean algorithm. The last two rows, which are easily calculated from the previous ones according to equation (D2), provide the values $x_{i}, y_{i}$. We have obtained $x=-7$ and $y=12$. Since we wish a value $0 \leq x<N$ and $0 \leq y<n$, we must correct the equation as $n(x+N)+N(y-n)=1$, so that, comparing with equation (46), we get $x=\llbracket M \rrbracket+1=24$ and $M=41$, hence $m=12$. Since $M>m$, the cyclic scale previous to $n=53$ is $n=41$.

\footnotetext{
${ }^{20}$ For $i \geq 2$, these variables are equivalent to duplicate equation (D1), dividing it by $a$ and $b$, and defining $x_{i}=\frac{d_{i}}{a}$, $y_{i}=\frac{d_{i}}{b}$. As $x_{1}=y_{0}=0$, the new variables are integers.
} 


\section{Acknowledgements}

I would like to thank the Editors Emmanuel Amiot, Jason Yust and, in particular, Thomas Fiore and Clifton Callender for valuable remarks and comments. Clifton Callender pointed out the existence of a similar algorithm (Carey 1998) for obtaining well-formed scales. I would also like to thank several anonymous referees for their thorough revision of this paper or earlier versions thereof, as well as pointing out crosslinks, helpful discussions, hints, and comments.

\section{References}

Berstel, Jean, and Aldo de Luca. 1997. "Sturmian Words, Lyndon Words and Trees." Theor. Comput. Sci. 178: 171-203.

Calkin, Neil, and Herbert S. Wilf. 2000. "Recounting the Rationals." Amer. Math. Montly 107: 360-363.

Carey, Norman. 1998. "Distribution Modulo 1 and Musical Scales." Ph.d. dissertation, Rochester NY.

Carey, Norman, and David Clampitt. 1989. "Aspects of Well-Formed Scales." Music Theory Spectrum 11 (2): $187-206$.

Carey, Norman, and David Clampitt. 1996. "Self-similar Pitch Structures, their Duals, and Rythmic Analogues." Perspectives of New Music 34 (2): 62-87.

Carey, Norman, and David Clampitt. 2012. "Two Theorems Concerning Rational Approximations." Journal of Mathematics and Music 6 (1): 61-66.

Carey, Norman, and David Clampitt. 2017. "Addendum to "Two Theorems Concerning Rational Approximations"." Journal of Mathematics and Music DOI:10.1080/17459737.2017.1286761.

Clampitt, David, and Thomas Noll. 2011. "Modes, the Height-Width Duality, and Handschin's Tone Character." Music Theory Online 17 (1).

Clough, John, and Jack Douthett. 1991. "Maximally Even Sets." J. Music Theory 35: 93-173.

Clough, John, and Gerald Myerson. 1985. "Variety and Multiplicity in Diatonic Systems." J. Music Theory 29: $249-270$.

Clough, John, and Gerald Myerson. 1986. "Musical Scales and the Generalized Circle of Fifths." Am. Math. Monthly 93: 695-701.

Douthett, Jack, and Richard Krantz. 2007. "Continued Fractions, Best Measurements, and Musical Scales and Intervals." Journal of Mathematics and Music 1 (1): 47-70.

Farey, John. 1816. "On a Curious Property of Vulgar Fractions." Phil. Mag. 47: 385-386.

Gibbons, Jeremy, David Lester, and Richard Bird. 2006. "Funtional Pearl: Enumerating the Rationals." J. Funct. Program. 16 (3): $281-291$.

Graham, Ronald L., Donald E. Knuth, and Oren Patashnik. 1994. Concrete Mathematics: A Foundation for Computer Science. 2nd ed. Boston, MA, USA: Addison-Wesley Longman Publishing Co., Inc.

Hall, Rachel W., and Krešimir Josić. 2001. "The Mathematics of Musical Instruments." American Mathematical Monthly 108 (4): 347-357.

Hellegouarch, Yves. 1999. "Gammes Naturelles." Gazette des Mathématiciens 81: 25-39, 82: 13-26.

Jedrzejewski, Franck. 2008. "Generalized Diatonic Scales." Journal of Mathematics and Music 2 (1): 21-36.

Jedrzejewski, Franck. 2009. Pseudo-diatonic Scales. Vol. 37. Berlin, Heidelberg: Springer.

Kassel, Adrien, and Christian Kassel. 2010. "On Hellegouarch's Definition of Musical Scales." Journal of Mathematics and Music 4 (1): 31-43.

Khinchin, A. Ya. 1964. Continued Fractions. Chicago: University of Chicago.

Kolman, Oren. 2004. "Transfer Principles for Generalized Interval Systems." Perspectives of New Music 42 (1): 150-190.

Krantz, Richard, and Jack Douthett. 2011. "Algorithmic and Computational Approaches to Pure-tone Approximations of Equal-tempered Musical Scales." Journal of Mathematics and Music 5 (3): 171-194.

Lewin, David. 1987. Generalized Musical Intervals and Transformations. New Haven: Yale University Press.

Liern, Vicente. 2015. "On the Construction, Comparison, and Exchangeability of Tuning Systems." Journal of Mathematics and Music 9 (3): 197-213.

Lothaire, M. 2002. Algebraic Combinatorics on Words. Cambridge: Cambridge University Press.

Loya, Paul. 2017. Amazing and Aesthetic Aspects of Analysis. New York: Springer. Available online: Course in Undergraduate Analysis (2006). Department of Mathematics, Binghamton University, Binghamton NY, http://www . math. binghamt on. edu/dennis/478.f07/EleAna.pdf.

Noll, Thomas. 2006. Facts and Counterfacts: Mathematical Contributions to Music-theoretical Knowledge. Berlin: W\&T Verlag.

Noll, Thomas. 2007. "Musical Intervals And Special Linear Transformations." Journal of Mathematics and Music 